\title{
Integrative Molecular and Clinical Profiling of Acral Melanoma Identifies LZTR1 as a Key Tumor Promoter and Therapeutic Target
}

Ruth Halaban ( $\nabla$ ruth.halaban@yale.edu )

Yale University School of Medicine https://orcid.org/0000-0001-8451-1964

Aaron Newman

Stanford University https://orcid.org/0000-0002-1857-8172

Farshad Farshidfar

Institute for Stem Cell Biology and Regenerative Medicine, Stanford University,

Cong Peng

Xiangya Hospital, Central South University

Chaya Levovitz

IBM (United States)

James Knight

Yale University https://orcid.org/0000-0003-1166-0437

Antonella Bacchiocchi

Yale University

Juan Su

Xiangya Hospital, Central South University

Kahn Rhrissorrakrai

IBM (United States)

Mingzhu Yin

Yale University School of Medicine

Mario Sznol

Yale University

Stephan Ariyan

Yale University

James Clune

Yale University School of Medicine

Kelly Olino

Yale University School of Medicine

\section{Laxmi Parida}

IBM Research - Thomas J. Watson Research Center https://orcid.org/0000-0002-7872-5074 Joerg Nikolaus 
Yale University School of Medicine

\section{Meiling Zhang}

Yale University School of Medicine

\section{Shuang Zhao}

Xiangya Hospital, Central South University

\section{Yan Wang}

Department of Dermatologic Surgery Institute of Dermatology, Chinese Academy of Medical Sciences \& Peking Union Medical College

\section{Gang Huang}

Department of Bone and Soft Tissue oncology, Hunan Cancer Hospital, Affiliated Tumor Hospital of Xiangya Medical School of Central South University

\section{Miaojian Wan}

Department of Dermatology, The Third Affiliated Hospital, Sun Yat-sen University

\section{Xianan Li}

Department of Bone and Soft Tissue oncology, Hunan Cancer Hospital, Affiliated Tumor Hospital of Xiangya Medical School of Central South University

\section{Jian Cao}

Rutgers Cancer Institute of New Jersey and the Department of Medicine, Robert Wood Johnson Medical School, Rutgers University

\section{Qin Yan}

Yale University https://orcid.org/0000-0003-4077-453X

\section{Xiang Chen}

Department of Dermatology, Xiangya Hospital, Central South University, Changsha 410008 https://orcid.org/0000-0001-8187-636X

\section{Article}

Keywords: cytoband chr22q11.21, oncogene, metastasis

Posted Date: September 30th, 2021

DOl: https://doi.org/10.21203/rs.3.rs-110475/v1

License: (c) (i) This work is licensed under a Creative Commons Attribution 4.0 International License. Read Full License

Version of Record: A version of this preprint was published at Nature Communications on February 23rd, 2022. See the published version at https://doi.org/10.1038/s41467-022-28566-4. 


\section{Integrative Molecular and Clinical Profiling of Acral Melanoma Identifies LZTR1 as a Key Tumor Promoter and Therapeutic Target}

Farshad Farshidfar ${ }^{1,2,14, \dagger}$, Cong Peng ${ }^{3, \dagger}$, Chaya Levovitz ${ }^{4}$, James Knight ${ }^{5}$, Antonella Bacchiocchi ${ }^{6}$, Juan $\mathrm{Su}^{3}$, Kahn Rhrissorrakrai ${ }^{4}$, Mingzhu Yin $^{3,7}$, Mario Sznol ${ }^{8}$, Stephan Ariyan $^{9}$, James Clune ${ }^{9}$, Kelly Olino ${ }^{9}$, Laxmi Parida ${ }^{4}$, Joerg Nikolaus ${ }^{10}$, Meiling Zhang ${ }^{7}$, Shuang Zhao ${ }^{3}$, Yan Wang ${ }^{11}$, Gang Huang ${ }^{12}$, Miaojian Wan ${ }^{13}$, Xianan $\mathrm{Li}^{12}$, Jian $\mathrm{Cao}^{7,15}$, Qin Yan ${ }^{7}$, Xiang Chen ${ }^{3, *}$, Aaron M. Newman ${ }^{1,2,{ }^{*}}$ and Ruth Halaban ${ }^{6,{ }^{*}}$

1 Institute for Stem Cell Biology and Regenerative Medicine, Stanford University, Stanford, CA, USA.

2 Department of Biomedical Data Science, Stanford University, Stanford, CA, USA.

3 Xiangya Hospital, Central South University, Changsha, China.

4 IBM Research, Yorktown Heights, NY, USA.

5 Yale Center for Genome Analysis, Yale University, New Haven, CT, 06520, USA.

6 Department of Dermatology, Yale University School of Medicine, New Haven, CT, USA.

7 Department of Pathology, Yale University School of Medicine, New Haven, CT, USA.

8 Department of Internal Medicine, Section of Medical Oncology, Yale University School of Medicine, New Haven, CT, USA.

9 Department of Surgery, Yale University School of Medicine, New Haven, CT, USA.

${ }^{10}$ Department of Molecular and Cellular Physiology, Yale University School of Medicine, New Haven, CT, USA.

11 Department of Dermatologic Surgery Institute of Dermatology, Chinese Academy of Medical Sciences \& Peking Union Medical College, Nanjing, China.

12 Department of Bone and Soft Tissue oncology, Hunan Cancer Hospital, Affiliated Tumor Hospital of Xiangya Medical School of Central South University, Changsha, Hunan, China.

13 Department of Dermatology, The Third Affiliated Hospital, Sun Yat-sen University, Guangzhou, China.

14 Current address: Tenaya Therapeutics, South San Francisco, CA, USA.

${ }^{15}$ Current address: Rutgers Cancer Institute of New Jersey and the Department of Medicine, Robert Wood Johnson Medical School, Rutgers University, New Brunswick, NJ, USA.

†These authors contributed equally.

${ }^{*}$ Corresponding authors:

Xiang Chen, Xiangya Hospital, Central South University, Changsha, China. Phone:

01186-731-84327303; E-mail: chenxiangck@126.com

Aaron M. Newman, Institute for Stem Cell Biology and Regenerative Medicine, Stanford University, Stanford, CA, USA. Phone: 650 724-7270; E-mail: amnewman@stanford.edu

Ruth Halaban, Department of Dermatology, Yale University School of Medicine, New Haven, CT, USA. Phone: 203 785-4352; E-mail: ruth.halaban@yale.edu 


\begin{abstract}
Acral melanoma, the most common melanoma subtype among non-Caucasian individuals, is associated with poor prognosis. However, its key molecular drivers remain obscure. Here, we performed integrative genomic and clinical profiling of acral melanomas from a cohort of 104 patients treated in North America or China. We found that recurrent, late-arising amplifications of cytoband chr22q11.21 are a leading determinant of inferior survival, strongly associated with metastasis, and linked to downregulation of immunomodulatory genes associated with response to immune checkpoint blockade. Unexpectedly, LZTR1 - a known tumor suppressor in other cancers - is a key candidate oncogene in this cytoband. Silencing of LZTR1 in melanoma cell lines caused apoptotic cell death independent of major hotspot mutations or melanoma subtypes. Conversely, overexpression of LZTR1 in normal human melanocytes initiated processes associated with metastasis, including anchorage-independent growth, formation of spheroids, and increased levels of MAPK and SRC activities. Our results provide new insights into the etiology of acral melanoma and implicate LZTR1 as a key tumor promoter and therapeutic target.
\end{abstract}




\section{INTRODUCTION}

Over the last two decades, a tremendous effort has been made to understand the genomic basis of melanoma. Collectively, these analyses have shown that sunexposed melanomas harbor a large number of mutations and genomic rearrangements associated with ultraviolet (UV) radiation ${ }^{1-6}$. In contrast, acral melanomas, originating from hairless skin such as palms and soles, display a lower mutational burden, a higher rate of structural alteration, and poorer survival outcomes ${ }^{2,4,7-17}$. BRAF and NRAS are the most frequently affected oncogenes in acral melanomas but at a lower frequency compared to sun-exposed melanomas, whereas KIT mutations are more common in acral melanomas ${ }^{14,18-23}$. Copy number variation $(\mathrm{CNV})$ is a well-established feature of acral melanomas, contributing to aberrant regulation of several pathways affecting cell proliferation and gene expression. These include amplification of CDK4, CCND1, MAPK1 and NOTCH2; loss of CDKN2A (p16 ${ }^{\mathrm{INK} 4}$ ) and NF1; inactivation of TP53; modifications of chromatin regulators (e.g., HDAC amplification and loss of $A R I D 1 A$ and $A R I D 1 B) ;$ and alterations in TERT $T^{11,13,14,20,23-25}$. Despite these findings, attempts to treat acral melanoma with targeted inhibitors, such as CDK4/6 inhibitors, have failed ${ }^{12}$.

While most genomic studies of acral melanoma have been limited to relatively small clinical cohorts ${ }^{1,9,13,26,27}$, a recent whole genome analysis of 87 patients $-90 \%$ of which were of European ancestry - further confirmed the importance of structural rearrangements and copy number aberrations in this disease ${ }^{15}$. Given the predominance of acral melanoma in non-Caucasian populations ${ }^{28,29}$ and the lack of effective targeted treatment options, large-scale genomic surveys of acral melanomas from ethnically diverse populations are needed.

Here, we applied whole exome (tumor/normal) and RNA sequencing to characterize acral melanomas from 104 patients treated in the United States $(n=37)$ and China $(n=67)$, most of whom had long-term follow-up data available. Through comparative genomic analysis with 157 sun-exposed melanomas, we identified novel molecular features of acral melanoma; generated the first prognostic map linking highly recurrent somatic aberrations in acral melanoma to risk of death; and found that latearising focal amplifications in chr22q11.21 are associated with lymph node involvement and distant metastasis, leading to poor outcomes. Within chr22q11.21, we identified LZTR1 - a known tumor suppressor in other cancers - as a key candidate driver of metastasis. Our findings reveal novel molecular insights of acral melanoma pathogenesis and designate LZTR1 as a new therapeutic target. 


\section{RESULTS}

\section{Genomic characteristics of acral and sun-exposed melanoma}

To characterize the genomic landscape of acral melanoma across ethnically diverse patient populations, we analyzed 104 tumors, including 97 by whole exome sequencing (WES), from patients treated in North America ('Yale') or China ('CSU') (Table 1). Both cohorts spanned all disease stages, included long-term follow-up, and encompassed patients with distinct ethnic origins, including Caucasian ( $n=31$; Yale) and Asian ancestry ( $n=67$; CSU) (Supplementary Fig. 1a and Supplementary Table 1). We also applied WES to profile 134 tumors from patients with sun-exposed melanoma, including patients with stage I through IV disease (Table 1). Notably, sunexposed patients showed longer survival time than acral melanoma patients, consistent with previous studies ${ }^{16,17}$ (Supplementary Fig. 1b). Peripheral blood leukocytes were analyzed as germline controls and whole-transcriptome sequencing (RNA-seq) was applied to 105 tumors, including 38 acral and 37 sun-exposed melanomas with matched WES data (Table 1 and Supplementary Table 1). Tumor purities, clinical follow-up, and median survival times were comparable between acral cohorts, supporting their combined assessment (Supplementary Fig. 1c,d; Supplementary Table 1).

To verify key somatic lesions in acral melanoma, we began by performing a comparative genomics analysis. We observed striking variation in the prevalence of single nucleotide variants (SNVs) and insertions/deletions (indels) between melanoma

subtypes, confirming a nearly ten-fold lower mutational burden in acral melanoma 2,13 (median of 406 vs. 42 nonsynonymous variants per exome in sun-exposed vs. acral melanoma, respectively; $P=2.2 \times 10^{-6}$, two-sided Wilcoxon rank sum test; Fig. 1a, Supplementary Table 2, Supplementary Data). The most commonly mutated genes in acral melanomas were RAS family members (22\% in NRAS, KRAS, and HRAS), followed by KIT (15\%), CGREF1 (10\%), BRAF (8\%), and TP53 (4\%). With the exception of CGREF1, which was limited to CSU patients, recurrence frequencies were similar between cohorts (Supplementary Table 2). Mutational signature analysis ${ }^{17}$ corroborated the prevalence of UV-induced mutagenesis in sun-exposed melanomas. In contrast, mutational signatures in acral melanomas were largely attributable to deamination of 5-methylcytosine (signature 1), which can arise from reactive oxygen species during melanin synthesis ${ }^{30}$, as well as alkylating agents (signature 11) and APOBEC activity (signature 13) (Fig. 1a, bottom, and Supplementary Data).

As expected, focal amplifications were a core feature of acral melanoma in both cohorts (median of 20 vs. 11 per exome in acral vs. sun-exposed melanoma, respectively; $P=1.24 \times 10^{-10}$, two-sided Wilcoxon rank sum test; Fig.1b, top; Supplementary Figs. 1e and 2a; Supplementary Table 3). Among highly recurrent gene-level amplifications with at least four copies, those in chromosomes 4, 5, 8, 11, 12, and 22 were nearly exclusive to acral melanomas in our study (Fig. 1c; Supplementary 
Table 3). The most common amplifications enriched in acral melanoma were in cytobands 11q13.3 (47\%), 5p15.33 (42\%), 8q24.3 (42\%), 22q13.1 (39\%), and 22q11.21 (38\%) (Fig. 1b; Supplementary Fig. 2a, Supplementary Table 3). Recurrent focal deletions, which included alterations in known genes such as CDKN2A (9p21.3) 22,31,32, were less prevalent than in sun-exposed cases (Fig.1b, bottom; Supplementary Fig. 2b, Supplementary Table 4). Amplification and deletion frequencies were largely maintained in both acral cohorts (Supplementary Tables 3 and 4). We also identified multiple fusion genes with potential roles in oncogenesis, including several not previously described in acral melanoma (Supplementary Table 5).

Collectively, these results provide a comprehensive resource of somatic lesions in acral melanomas from genetically-distinct patient populations; corroborate and extend previous genomic studies ${ }^{2,4,7-15}$, and demonstrate the integrity and high quality of our data for downstream clinical analysis.

\section{Somatic determinants of risk in acral melanoma}

Having systematically cataloged somatic aberrations in nearly 100 acral melanomas, we next sought to evaluate their clinical significance. We began by focusing on amplification events owing to their unique prevalence in this disease (Fig. 1b). Starting with the most statistically-significant peaks detected by GISTIC 33 in a pooled analysis of both acral cohorts $\left(Q<10^{-5}\right)$, we identified several loci linked to adverse overall survival, including peaks involving cytobands 22q11.21 and 22q13.1. Among them, cytoband 22q11.21 was most strongly associated with inferior overall survival (adjusted $P<0.05$, univariate Cox regression of time from tumor resection; Supplementary Fig. 3a, Supplementary Table 6). This result was maintained when expanding the analysis to include all focal events identified by GISTIC $(Q<0.05)$ with at least $10 \%$ recurrence frequency in both acral cohorts and all genes with a nonsynonymous mutation frequency of at least $5 \%$ in either melanoma subtype (Fig. 2a; Supplementary Table 6). We also considered focal amplifications identified from the largest cohort (CSU) and tested in each cohort separately (Fig. 2b; Supplementary Fig. 3b). Again, 22q11.21 amplification was a leading determinant of adverse survival.

Given this observation, we sought to better understand 22q11.21 focal amplification and the factors underlying its clinical phenotype. We first tested whether $22 q 11.21$ is a surrogate for advanced disease at the time of tumor resection. Intriguingly, 22q11.21 amplifications were observed across all stages except stage I disease ( $P=0.03$, Chi $X^{2}$ test; Fig. 2c, left). We verified this result in three independent acral melanoma cohorts, including an external dataset comprised of 33 patients for whom stage at presentation was known ${ }^{11}$, demonstrating that 22q11.21 amplification is a recurrent late-arising event in acral melanoma (Supplementary Fig. 3c). Given this result, we reassessed survival associations using stage as a covariate. Regardless of 
whether we examined all patients or just those with stage II through IV disease, 22q11.21 amplifications remained significant after multivariate adjustment for stage ( $P=$ 0.008 and 0.024 , respectively; Cox proportional hazards regression). This was also true for acral patients with advanced disease (III or IV) $(P=0.03$, Cox proportional hazards regression; Supplementary Fig. 3c), for whom stage alone did not significantly stratify outcomes.

As a common late-arising event, we next tested if 22q11.21 amplifications might correlate with tumor progression. Indeed, in both acral cohorts, we observed a strong positive correlation between 22q11.21 amplification frequency and the number of positive lymph nodes (Fig. 2c, right; Supplementary Fig. 3e). Remarkably, nearly $75 \%$ of patients with $>1$ positive lymph node harbored at least one gain of 22q11.21 (Fig. 2c, right). Reanalysis of WES data from an independent study ${ }^{11}$ confirmed this trend (Supplementary Fig. 3f). While this association was observed in both primary and metastatic tumor specimens, the latter showed a modest but consistent increase in amplification frequency after controlling for lymph node status (Supplementary Fig. $\mathbf{3 g}$ ). No other associations with clinical indices were observed (Supplementary Fig. 3h, Supplementary Table 1).

Taken together, these data reveal that $22 q 11.21$ focal amplification is a conserved, late-arising somatic event linked to poor survival and regional metastasis in acral melanoma, independent of Caucasian or Asian ancestry. Accordingly, this event could represent a critical step in the initiation or maintenance of nodal metastasis.

\section{Integrative genomics of 22q11.21 focal amplification}

To understand the biological significance of 22q11.21 amplification in acral melanoma, we next examined transcriptional hallmarks of 22q11.21-amplified tumors. By employing a linear model adjusted for stage (Methods), we rank-ordered genes by their differential expression in 22q11.21-amplified tumors and performed gene set enrichment analysis ${ }^{34}$ (Fig. 2d). Overall, 22q11.21-amplified melanomas were significantly enriched in canonical signaling pathways associated with tumorigenesis and metabolic activity, including MYC target genes, oxidative phosphorylation, and unfolded protein response ${ }^{35}$. In contrast, patients with non-amplified tumors showed higher expression of immunoreactive programs such as IL6/JAK/STAT and IFN-Y response pathways. We hypothesized that such patients might be superior candidates for existing or emerging immunotherapies (Fig. 2d). Consistent with this possibility, we observed a striking reciprocal relationship between 22q11.21-amplification and the expression of immunomodulatory genes, including key targets of immune checkpoint blockade (e.g., PDCD1, CTLA4) (Fig. 2e). Among patients with high expression of immunomodulatory genes, only $12 \%$ were amplified, whereas among patients with low expression, 62\% were amplified (Fig. 2f). This result was highly significant $(P=0.009$, 
Fisher's exact test), indicating that 22q11.21-amplified and non-amplified tumors preferentially reflect "cold" and "hot" tumor microenvironments, respectively.

To extend these observations to single cells, we applied single-cell RNA sequencing (scRNA-seq) to an acral melanoma tumor specimen with four additional copies of 22q11.21, as determined by WES (Supplementary Fig. 4a, Supplementary Data). Using canonical marker genes and copy number inference via CONICSmat ${ }^{36}$, 321 single-cell transcriptomes were confidently identified as melanoma cells (Supplementary Fig. 4b, Methods). We confirmed over-expression of genes on the 22q arm, consistent with WES (Supplementary Fig. 4c). However, 22q expression levels were heterogeneous across cells, indicating variability in the number of copies per cell (Supplementary Fig. 4c). By dividing malignant cells into two groups according to the median expression of 22q arm genes (selected from within the 22q11.21 peak identified by GISTIC), we observed the same amplification-enriched pathways identified in bulk tumors, including oxidative phosphorylation and MYC targets, confirming their malignant origin (Supplementary Fig. 4d).

Immature cancer cells often display elevated metabolism via oxidative phosphorylation and MYC activity ${ }^{37}$ and stemness features in melanoma tumors have been linked to poor survival ${ }^{38-41}$. To test whether 22q11.21-amplified cells exhibit an immature cellular phenotype, we employed CytoTRACE, a recently described in silico method for predicting developmental potential on the basis of single-cell transcriptional diversity ${ }^{42}$. Indeed, cells with higher relative copies of 22q11.21 were predicted to be less mature (Fig. 2g). Notably, this result was independent of genes physically located on 22q, implying that 22q11.21-amplified cells exhibit a more accessible genome, a hallmark of immature cells in normal tissues ${ }^{42}$ (Supplementary Fig. 4e).

Finally, we leveraged the $t$-statistic to rank genes in $22 q 11.21$ according to their expression in amplified versus non-amplified tumors (Fig. $2 \mathrm{~h}$, Supplementary Table 7 ). The top-ranking gene associated with amplification was LZTR1 (leucine zipper like transcription regulator 1). We were struck by this result because LZTR1, a member of the Kelch-like (KLHL) family and an adaptor for Cullin 3 (CUL3) ubiquitin ligase complexes ${ }^{43,44}$, is considered a tumor suppressor in schwannoma and glioblastoma ${ }^{43,45,46}$. Nevertheless, we found that high expression of LZTR1 is predictive of poor outcome, both in acral and sun-exposed melanomas from this study, and in 443 advanced sun-exposed melanomas profiled by TCGA (The Cancer Genome Atlas) (Supplementary Fig. 5, Methods). Beyond LZTR1, we noted that ZNF74, a zinc finger protein, and CRKL (CRK like proto-oncogene, adaptor protein), a recurrently amplified gene in multiple carcinomas ${ }^{47-50}$, including non-small cell lung cancer $(3 \%-13 \%$ of cases $)^{47-49}$, were ranked $2^{\text {nd }}$ and $3^{\text {rd }}$ in our analysis, respectively. Given these results, we set out to characterize the biological functions of these genes to determine which, if any, underlie the observed clinical phenotype of 22q11.21 amplification. 


\section{Suppression of LZTR1 attenuates melanoma cell proliferation and induces apoptosis independent of Ras or MAPK activity}

We began by silencing several chr22q11.21-amplified genes using lentiviral delivery of short hairpin RNAs (shRNAs) (Supplementary Table 8), with the goal of determining the impact of targeted knockdowns on melanoma cell proliferation. Treatment of two acral melanoma cell lines with ZNF74 shRNA had a modest effect on cell proliferation (Supplementary Fig. 6a). Similarly, while downregulation of $C R K L$ induced growth arrest, only one of three shRNAs against $C R K L$ successfully downregulated $C R K L$, and only two of five tested cell lines were highly affected (Supplementary Fig. 6b). Conversely, silencing of LZTR1 consistently arrested cell proliferation. This was the case regardless of subtype (acral or sun-exposed) or mutations in BRAF or NRAS (Fig. 3a, b). In addition, we observed growth arrest in normal melanocytes derived from two independent foreskins (Fig. 3a, b). We ruled out off-target effects because six different LZTR1-directed shRNAs induced growth arrest, as did CRISPR-Cas9 sgRNA directed against LZTR1 (Fig. 3a-c; Supplementary Fig 5c; Supplementary Table 8). The observed phenotype had a long-term effect since LZTR1-null melanoma cells did not survive in vitro, whereas cells infected with control shRNA (scrambled) continued to proliferate. We also tested depletion of SNAP29 and THAP7, both of which are physically located on 22q11.21 but whose expression levels were not significantly linked to 22q11.21 amplification (Supplementary Table 7). Knockdown of these genes had little to no effect on proliferation (Supplementary Fig. 5d, e).

Given these results, we sought to better understand the biological consequences of LZTR1 knockdown. Inactivating germline mutations in LZTR1 are associated with Noonan syndrome and functional studies have linked LZTR1 inactivation to RAS ubiquitination, increased RAS-MAPK signaling, and cell proliferation ${ }^{51-56}$. Indeed, suppression of LZTR1 in melanoma cells increased the constitutive levels of GTPbound RAS, an effect similar to that observed in growth factor-stimulated cells ${ }^{54,57}$. RAS-GTP levels increased in NRAS- or BRAF-mutant melanoma cells without a change in total RAS protein (Fig. 4a, b).

We also observed widespread changes in MAPK signaling following LZTR1 knockdown. For example, there was an increase in pERK in melanoma cells carrying $B R A F^{V 600 E}, P D E 4 D I P-B R A F$, or GOLG4A-RAF1 (Fig. 4c). In contrast, pERK decreased in NRAS ${ }^{Q 61 L / R}$ melanoma cells, PDE8A-RAF1 fusion-bearing melanoma cells, and normal human melanocytes (Fig. 4c). ERK activation was likely due to an increase in BRAF levels (Fig. 4d), enhancing BRAF activity. Treatment of melanoma cells with BRAF $^{\mathrm{V} 600 E / K}$ or pan-RAF inhibitors (PLX4032 or LY3009120) reduced shLZTR1-induced pERK activation (Fig. 4e), rendering further support for the role of BRAF kinase activity. 
On the other hand, ERK inhibition in shLZTR1-treated cells could potentially arise from RAS translocation to the cytoplasm (Fig. 4f), and the consequent disassociation from its membrane-bound mitogenic effectors, which are critical for NRAS ${ }^{Q 61 / L R}$ mutant and WT cells lacking BRAF mutations. RAS translocation was not linked to de-ubiquitination, because loss of LZTR1 did not change the levels of ubiquitinated RAS (Supplementary Fig. 7a). RAF1 levels were diminished in most cell lines (Fig. 4d), reflecting a decrease in gene expression. Thus, downregulation of LZTR1 induces growth arrest independently of ERK activity, the presence of BRAF or NRAS oncogenes, and changes in RAS-GTP levels.

We next explored if our in vitro melanoma systems effectively recapitulate key 22q11.21-related signaling pathways observed in vivo. To this end, we performed bulk RNA-sequencing of a melanoma cell line (YUSIK) to assess the impact of LZTR1 knockdown. Remarkably, depletion of LZTR1 induced transcriptome-wide changes that largely mirrored those observed in bulk tumors and single melanoma cells (Fig. 5a).

We noticed that among altered transcriptional programs, apoptosis-related genes were elevated in cell lines and tumors with lower LZTR1 expression (Fig. 5a). These data are supported by an increase in caspase activity after treatment with LZTR1 shRNA or sgRNA (Fig. 5b, c), which led to the degradation of known caspase substrates $^{58}$, including pRb, p53, PARP1, NFKB, and GOLGA4 (Fig. 5d). Notably, GOLGA4 localizes to the Golgi apparatus, the subcellular site of LZTR1 ${ }^{59}$. Moreover, shLZTR1-induced caspase activity was suppressed by the pan-caspase inhibitor IDN6556 (Emricasan), which also rescued several substrates, including LZTR1 (Fig. 5c, d). These data are consistent with a previous report showing that LZTR1 undergoes caspase-mediated degradation ${ }^{59}$. Furthermore, shLZTR1 led to disruptions of cellular organization, including actin depolymerization into irregular shapes (Fig. 5e, left), or formation of actin rings around the Golgi and nucleus (Fig. 5e, right). Such changes are characteristic of cells undergoing fast or slow apoptotic death, respectively ${ }^{60}$.

Several cell cycle proteins were also downregulated, in line with pathway enrichment analyses (Fig. $5 f$ and Supplementary Fig. $\mathbf{7 b}-\mathbf{e}$ ). In addition, retinoblastoma proteins ( $\mathrm{pRb}$ and $\mathrm{p} 130$ ) were suppressed in the nine melanoma cell lines tested (Fig. 5f). This was likely due to ubiquitination and degradation ${ }^{61}$, as $p R b$ was not rescued by the caspase inhibitor IDN-6556 (Fig. 5d). Elimination of pRb may enhance mitochondrial-mediated apoptosis because it leads to reduced mitochondrial mass, reduced activity of the electron transport chain, and increased reactive oxygen species (ROS) $)^{62-65}$.

A major reason for growth arrest in some melanoma cell lines is downregulation of MITF, a lineage-specific transcription factor critical for melanocyte and melanoma cell proliferation ${ }^{66}$. MITF stability is reduced when phosphorylated by MAPK or $\mathrm{KIT}^{67,68}$, and this process was clearly observed in three out of four melanoma cell lines with 
increased ERK activity (Fig. 5g, as compared to Fig. 4c, e). Downregulation of MITF, as expected, is associated with decrease in tyrosinase (TYR), the key enzyme in melanin synthesis as well as cellular pigmentation (Fig. $5 \mathbf{h}, \mathbf{i})$. These results are consistent with our published observations using the same melanoma cell lines ${ }^{69}$.

\section{Overexpression of LZTR1 in normal melanocytes confers properties of malignant transformation and metastasis}

We next evaluated the impact of overexpressing LZTR1 in normal melanocytes and compared the effects to overexpression of CRKL. The latter is a SH3/SH2 adaptor protein that promotes lung cancer cell invasion via ERK activation ${ }^{70}$ and epithelialmesenchymal transition (EMT) in colorectal and pancreatic carcinomas ${ }^{71}$. Early passage human melanocytes (passage 4) were transduced with HA-tagged LZTR1 cloned into the plnd20 lentiviral vector, V5-tagged CRKL inserted into the PLX304 vector, or both constructs. Over-expression of these genes did not enhance the rate of cell proliferation; rather, melanocytes overexpressing CRKL grew slower compared to parental cells (Supplementary Fig. 8a). Nevertheless, within 2-3 days after infection, we noticed a striking induction of anchorage-independent growth, observed as cells overexpressing LZTR1 or CRKL formed three-dimensional clusters in 2D and 3D collagen cultures (Fig. 6a, top and bottom rows, respectively). Moreover, this result which was reminiscent of a malignant cell phenotype ${ }^{72}$ - was further enhanced when both genes were co-expressed (LZTR1+CRKL), leading to the formation of spheroids that detached from the surface of the dish (Fig. 6a).

During metastasis, primary melanoma cells detach from the dermis and migrate to secondary sites through increased cell-cell interactions and promotion of cancer cell survival. We therefore examined changes in adhesion proteins affecting cell-matrix and cell-cell interactions known to mediate aggregation, the formation of spheroids ${ }^{72}$, and in vivo $\mathrm{EMT}^{73,74}$. Our data show that $\mathrm{E}$-cadherin was downregulated whereas $\mathrm{N}$-cadherin and integrin $\beta 1$ were upregulated in response to increased expression of LZTR1 and CRKL, a process that was enhanced when both genes were co-expressed (Fig. 6b). Notably, our results with CRKL were consistent with HCT116 colon cancer cells, in which loss of CRKL was found to increase E-cadherin expression and shift the cells toward an epithelial phenotype ${ }^{71}$. Importantly, LZTR1 and CRKL, both alone and in combination, induced high levels of constitutively active ERK and SRC relative to parental cells (Fig. 6b, pERK and pSRC), functions that support viability and proliferation. We also identified downregulation of MITF in cells overexpressing CRKL as the possible cause for growth rate attenuation (Fig. 6b; Supplementary Fig. 8a). Consistent with this finding, while higher expression of MITF defines a proliferative subtype of melanoma (MITF ${ }^{\text {high }}-A X L^{\text {low }}$ ), lower expression is preferentially associated with invasion (MITFlow_AXL $\left.{ }^{\text {high }}\right)^{75}$. 
Based on these findings, we hypothesized that co-amplification of LZTR1 and $\mathrm{CRKL}$ might lead to increased rates of distant recurrence. Given that 22q11.21 amplification is a late-arising event in acral melanoma (Fig. 2c, left), we tested this hypothesis by examining acral melanoma patients diagnosed with stage II or III disease. Indeed, in patients for whom distant metastasis-free survival (DMFS) data were available, focal amplification of 22q11.21 was associated with earlier development of distant metastatic disease, with a median lead time of nearly 1 year (Fig. 6c).

Finally, we investigated whether overexpression of LZTR1 or CRKL release normal human melanocytes from their dependency on growth factors, a common phenotype of metastatic melanoma cells ${ }^{76}$. While normal human melanocytes retained their growth factor dependency (Supplementary Fig. 8), LZTR1, but not CRKL, enabled immortalized mouse melanocytes to form colonies and divide in the absence of their only required growth factor, TPA (tetradecanoyl phorbol acetate) $)^{77}$ (Fig. 6d,e). This phenotype is likely the consequence of MAPK activation, as seen by the presence of phosphorylated ERK (Fig. 6f).

Taken together, these results strongly implicate LZTR1 and CRKL in malignant transformation and the initiation of metastasis. While both genes showed similar phenotypes, the effects of overexpression were notably enhanced when LZTR1 and CRKL were co-expressed. However, only LZTR1 released immortalized mouse melanocytes from their dependency on growth factor, a characteristic shared by melanoma cells.

\section{DISCUSSION}

Acral melanoma has high incidence among non-Caucasian populations, accounting for up to $86 \%$ of melanomas diagnosed in Asian patients as compared to $\sim 10 \%$ of Caucasians ${ }^{29,78-84}$. Our work establishes common features of acral melanomas in cohorts from Asian and Caucasian populations. These include 1) the consistent association between specific focal amplifications and poor outcomes, and 2) the identification of LZTR1 as a key gene within 22q11.21, the most prognostic recurrent alteration identified in both acral cohorts. Based on these findings, we performed a comprehensive analysis of LZTR1 signaling pathways and obtained functional evidence for LZTR1 as a tumor promoter.

LZTR1 is co-amplified with CRKL and downregulation of each gene inhibits melanoma cell proliferation, albeit to varying degrees. While CRKL has been linked to tumor growth as a candidate oncogene in several human malignancies, including lung adenocarcinoma ${ }^{47-49}$, LZTR1 is generally considered a tumor suppressor. Germline mutations in LZTR1 are involved in Noonan syndrome ${ }^{53,85}$, schwannomatosis ${ }^{46}$ and glioblastoma ${ }^{43,86}$. Moreover, somatic loss-of-function mutations in LZTR1 occur in $22 \%$ 
of glioblastomas. These mutations drive self-renewal and growth of glioma spheres ${ }^{43}$, consistent with a role in tumor suppression. However, despite these findings, LZTR1 is amplified in a subset of carcinomas (up to $8.3 \%$ ), including bladder, uterine, and lung cancers $^{87,88}$. These data, coupled with our results, suggest that LZTR1 could have tumor-promoting capabilities in multiple human malignancies.

Unique aspects of our study include the broad range of tumor specimens analyzed and the utilization of cells harboring different oncogenes that modulate LZTR1 activity. For example, in NRAS-mutant melanoma, RAS mis-localized to the cytoplasm in response to shLZTR1 and caused MAPK inhibition. On the other hand, elimination of LZTR1 in BRAF-mutant cells increased BRAF levels, leading to ERK activation. In several cell lines, ERK activation induced growth arrest via MITF degradation, a process unique to melanocytes and the melanoma system ${ }^{67,68}$.

Importantly, our study demonstrates that LZTR1 and CRKL - two of the top three genes associated with ch22q11.2 amplification in acral melanoma - facilitate anchorage-independent growth in normal human melanocytes, likely by reducing $\mathrm{E}$ cadherin, increasing $\mathrm{N}$-cadherin, and activating integrin $\beta 1$. The reciprocal expression of $\mathrm{E}$-cadherin and $\mathrm{N}$-cadherin in early melanoma progression has been known for about two decades ${ }^{73,89}$, but to our knowledge, our findings link these events to genomic modification of two specific genes for the first time. In addition, we observed activation of MAPK and SRC kinases, the likely consequences of integrin signaling ${ }^{90,91}$. The ability of LZTR1 to convert immortalized mouse melanocytes to a growth-factor independent mode of proliferation, a major characteristic of melanoma cells in culture, further underscores its tumorigenic potential. These results agree with our genomic observation that ch22q11.21 amplification is a late-arising event associated with regional and distant metastasis.

Separately, we identified a striking inverse relationship between immunomodulatory genes and 22q11.21 amplification. It is tempting to speculate that high levels of LZTR1 reduce the inflammatory response while protecting cells from stress-induced apoptosis, thereby facilitating metastasis. Conversely, patients with low levels of LZTR1 preferentially harbor a hot tumor microenvironment, which might provide benefit from immunotherapy. Future studies will be needed to explore these possibilities.

In summary, we demonstrate that late-arising focal amplifications of cytoband 22q11.21 are a leading determinant of shorter survival time in acral melanoma. Our genomic and functional experiments provide critical new insights into the pathogenesis of this disease and strongly implicate LZTR1 as a novel tumor promoter and promising therapeutic target. 


\section{Acknowledgements}

This work was supported by the Melanoma Research Alliance (R.H., Q.Y., J.C.), the Sokoloff-MRA Award (R.H.), the Yale SPORE in Skin Cancer (Bosenberg and Kluger), the Roslyn and Jeremy Meyer Award (R.H.), the National Cancer Institute (A.M.N., R00CA187192), the Stinehart-Reed foundation (A.M.N.), the Stanford Bio-X Interdisciplinary Initiatives Seed Grants Program (IIP) (A.M.N.), the Virginia and D.K. Ludwig Fund for Cancer Research (A.M.N.), the Natural Science Foundation of China Major Projects of International Cooperation and Exchanges grant 81620108024 (X.C.), the General Program grant 81874138 (M.Y.), a New Investigator Award provided by Rutgers Cancer Institute of New Jersey (State of NJ appropriation and National Institutes of Health grant P30CA072720, J.C.), and a Melanoma Research Foundation Career Development Award (J.C.). We wish to acknowledge the Yale Center for Genome Analysis (YCGA) for performing WES and RNA-seq, David Calderwood and Ben Turk for providing the short hairpin RNA lentiviral vectors, the West Campus Imaging Core for confocal microscopy and cell imaging, Junkun Liu for performing cell immunostaining, Robert Straub and Jenna Ollodart for technical assistance. We thank for Dr. Doug Brash for his insight regarding acral melanoma mutations and to Zoe Halaban for her critical questions and enthusiastic support during these studies. We dedicate this manuscript to the memory of our colleague Dr. Deepak Narayan, a surgeon-scientist who provided great insight into this work and who will be remembered for his legacy of unparalleled innovation in the face of complex problems.

\section{Author Contributions}

A.M.N. and R.H. conceived the study, developed strategies for related experiments, and wrote the manuscript. X.C. initiated the collaborative studies, supervised sample collections from multi-clinical centers in China, exome and RNA-sequencing and some functional studies. F.F. co-wrote the paper and performed bioinformatics analyses with assistance from C.L., J.K., K.R., and L.P. R.H. performed key functional experiments with assistance from C.P., A.B., M.Y., M.Z., and J.N. A.B. performed experiments, tissue collection and processing (Yale cohort) and obtained clinical data. C.P. performed sample and clinical data collection and assisted with sequencing (CSU cohort). J.S. assisted with the collection of clinical data (CSU cohort). M.S. identified patients and collected clinical data (Yale cohort). S.A., D.N., J.C., K.O, S.Z., Y.W, G.H, M.W., and X.L contributed tumor specimens. J.C. and Q.Y. assisted with the conception of the study, performed experiments, and contributed to writing. All authors commented on the manuscript at all stages. A.M.N. and R.H. jointly supervised this work.

\section{Competing Interests}

The authors declare no competing interests. 

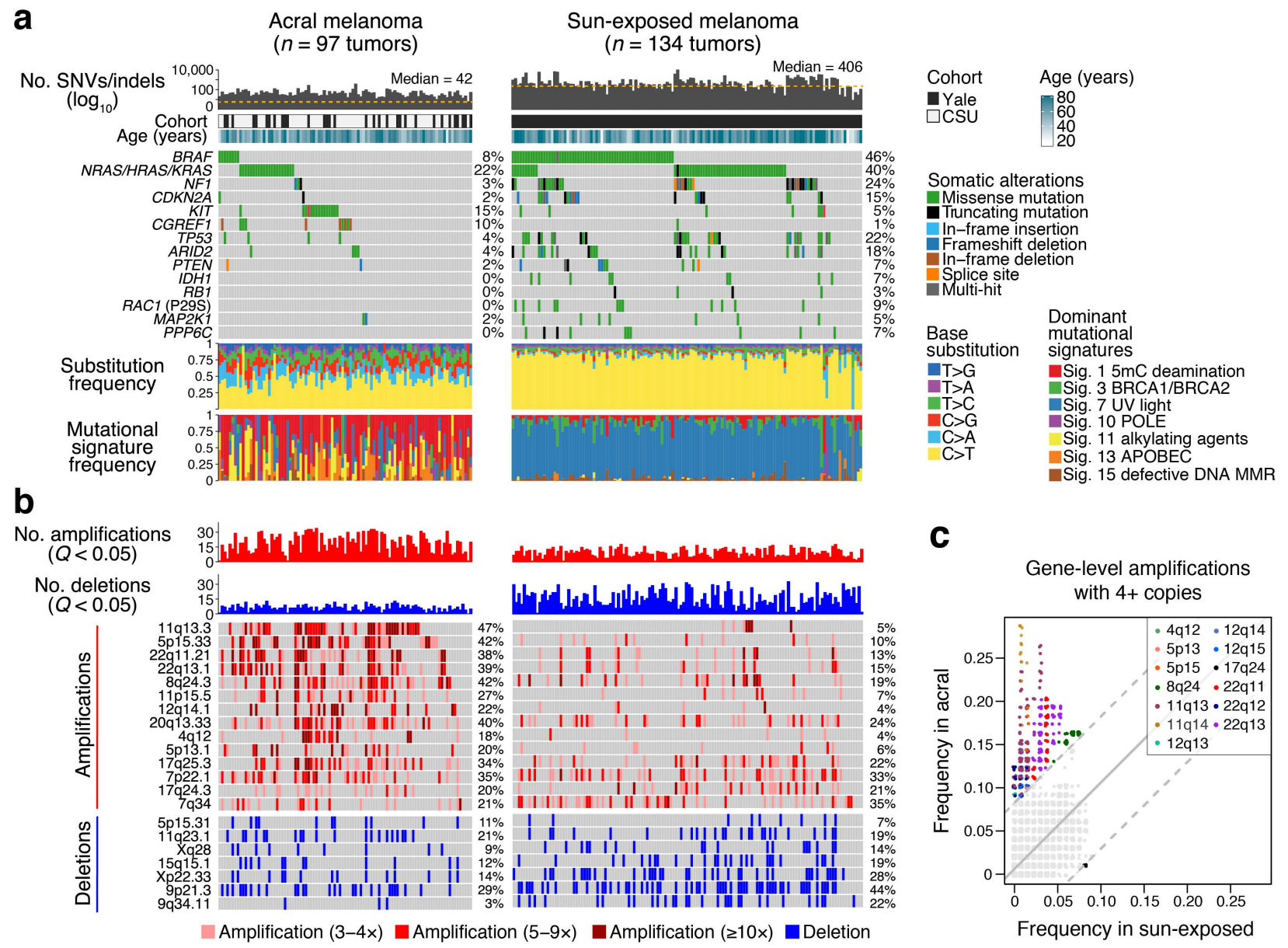

Figure 1: Landscape of somatic alterations in acral and sun-exposed melanomas. $\mathbf{a}-\mathbf{b}$, Genomic and clinical characterization of acral and sun-exposed melanoma samples sequenced in this work. a, The number of nonsynonymous SNVs and indels per melanoma exome (columns), cohort, age, frequently mutated genes (at least $5 \%$ recurrence frequency in either melanoma subtype), nonsynonymous base substitution frequencies, and dominant COSMIC mutational signatures ${ }^{92,93}$. Sig., signature; $5 \mathrm{mC}, 5-$ methylcytosine. b. The number of significant focal amplifications and deletions (GISTIC $Q<0.05$ ) per melanoma exome (columns), ordered identically to panel a. Cytobands with focal amplifications or deletions with at least $10 \%$ recurrence frequency in either melanoma subtype are shown (GISTIC $Q<10^{-5}$ ), ordered by the relative difference in recurrence frequency in acral versus sun-exposed melanoma. c, Genes are plotted according to the fraction of acral ( $y$-axis) or sun-exposed ( $x$-axis) tumors where they are present with 4+ copies. Considering the genome-wide distribution of differences in recurrence frequencies between melanoma subtypes, genes are identified as significantly recurrent in acral or sun-exposed melanomas if their $\mid z$-score $\mid \geq 3$ (dashed lines). Significantly recurrent genes are colored according to their cytoband location (inset). For clarity, a small amount of jitter was added to distinguish overlapping genes. 
a
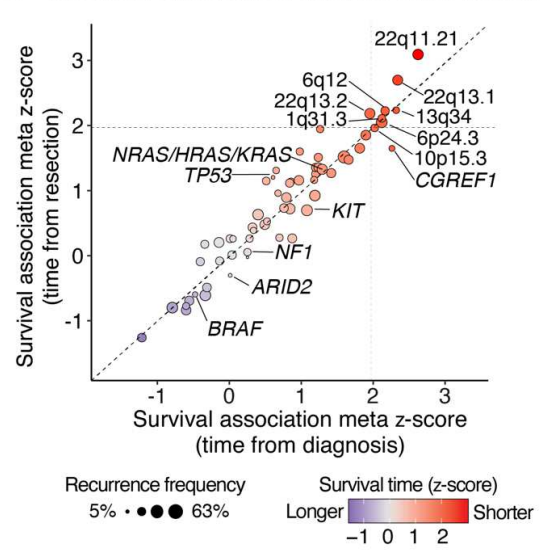

C

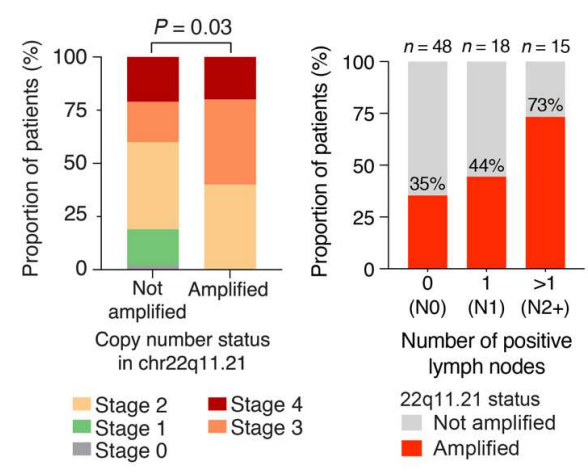

e

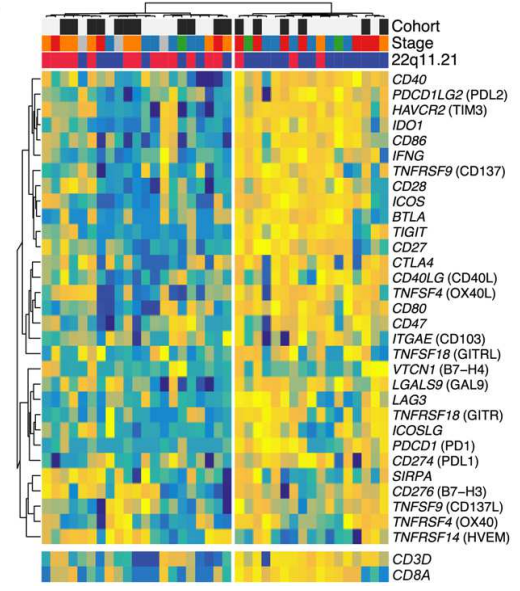

b

Association of 22q11.21 amplification with overall survival $+22 q 11.21$ Amplified $+22 q 11.21$ Not amplified
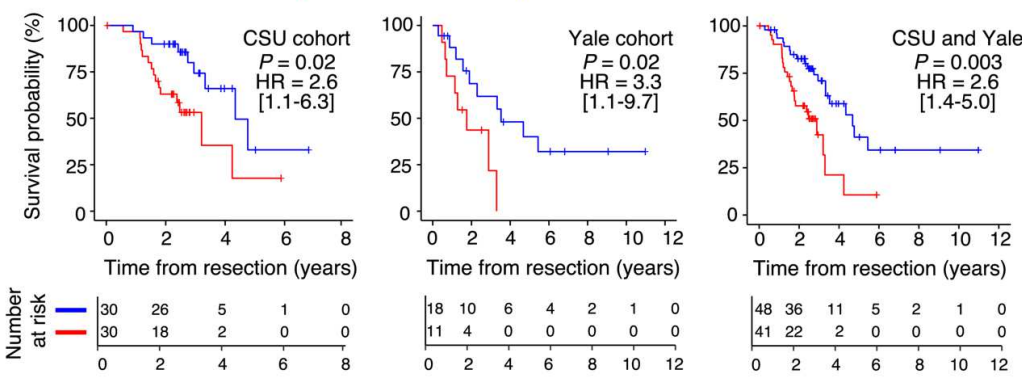

\begin{tabular}{|lllllll}
18 & 10 & 6 & 4 & 2 & 1 & 0
\end{tabular} $\begin{array}{ccccccc}11 & 4 & 0 & 0 & 0 & 0 & 0 \\ 0 & 2 & 4 & 6 & 8 & 10 & 12\end{array}$ $\begin{array}{lllllll}48 & 36 & 11 & 5 & 2 & 1 & 0\end{array}$

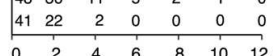

d chr22911.21 functional enrichments in acral melanoma

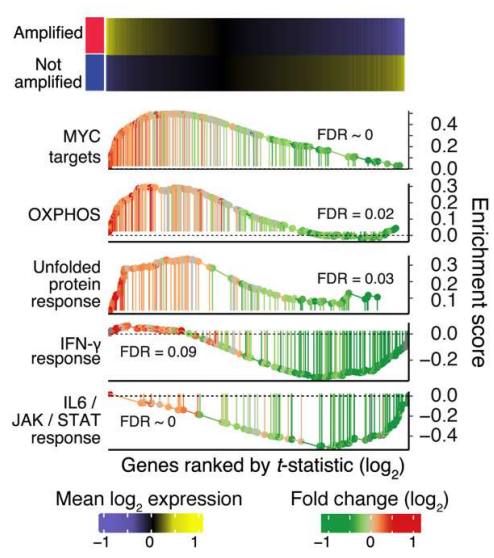

f

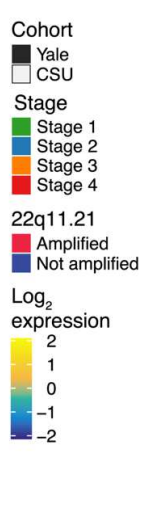

Immunomodulatory genes

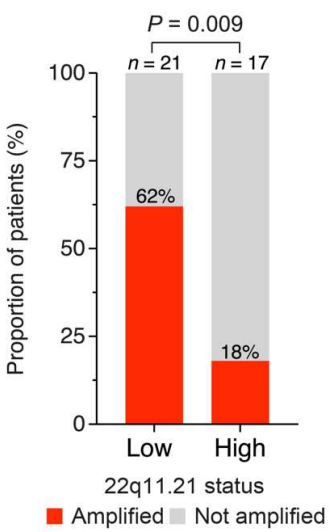

g
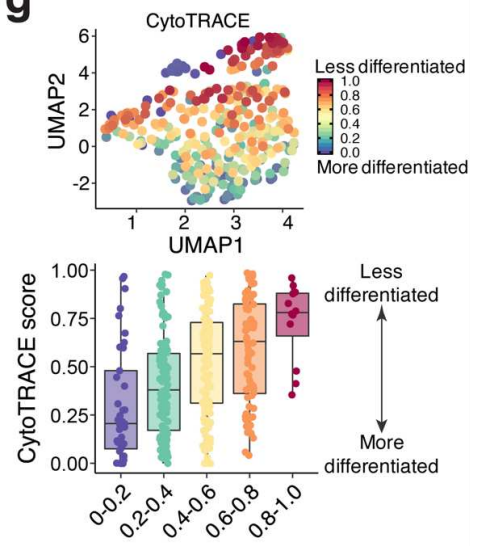

Normalized copies of 22q11.21 Less copies $\longleftrightarrow$ More copies

h Gene expression association with 22q11.21 amplification

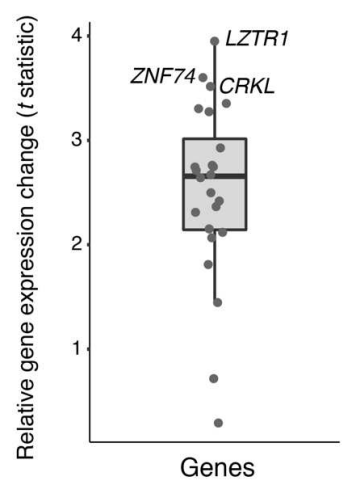

Figure 2: Focal amplifications in 22q11.21 are linked to shorter survival time, regional metastasis, and depletion of immunomodulatory programs in acral melanoma. a, Association between recurrent somatic alterations and overall survival (OS) in acral melanoma. Z-scores with positive and negative values indicated shorter and longer survival time, respectively $(|Z|>1.96$ is significant at $P<0.05$; Methods). OS was calculated from the date of diagnosis ( $x$-axis) and the date of tumor resection $(y$ axis). Shown are genes with a nonsynonymous mutation frequency of at least $5 \%$ in either melanoma subtype and focal copy number events with at least $10 \%$ recurrence frequency in each acral cohort (Supplementary Table 6). Focal amplifications in 
cytobands significantly associated with OS and recurrently mutated genes are labeled. Additional details are provided in Methods. b, Kaplan Meier curves showing overall survival of acral melanoma patients, stratified by 22.q11.21 amplification status and calculated from the date of tumor resection. Significance was assessed with the logrank test. HR, hazard ratio. 95\% HR confidence intervals are shown in brackets. c, Left: Acral melanoma tumor stage shown as a function of 22q11.21 amplification status. Statistical significance was evaluated by a Chi $X^{2}$ test. Right: Fraction of 22q11.21-amplified melanomas stratified by the number of involved lymph nodes ( $N$ stage). d, Hallmark signaling pathways significantly enriched in 22q11.21-amplified vs. non-amplified acral melanomas, as determined by pre-ranked Gene Set Enrichment Analysis (GSEA). Genes were ranked by $\log _{2}$ fold change adjusted for stage (Methods). OXPHOS, oxidative phosphorylation. e, Hierarchical clustering of 31 immunomodulatory genes (average linkage with Euclidean distance) in acral melanomas. CD3D and CD8A are included as lineage markers for T cells and CD8 T cells, respectively. $\mathbf{f}$, Bar plot showing frequency of 22q11.21-amplified acral melanomas separated into high and low immunomodulatory expression groups, as defined by clustering in panel e. Statistical significance was determined by Fisher's exact test. g, Single-cell differentiation status, as imputed by CytoTRACE ${ }^{42}$ (top), versus the estimated relative number of 22q11.21 copies per cell (normalized between 0 and 1), as imputed by CONICSmat ${ }^{36}$ (bottom), in cancer cells from a 22q11.21-amplified acral melanoma tumor (Supplementary Fig. 4; Methods). Single-cell transcriptomes are visualized in the top subpanel by Uniform Manifold Approximation and Projection (UMAP). $\mathbf{h}$, Association between gene expression and 22q11.21 amplification status in Yale and CSU acral melanoma cohorts. Only genes physically located on cytoband 22q11.21 are shown. Group comparisons were performed using a two-sided $t$-test with unequal variance. The top three genes are indicated. Amplified, 3+ copies. 
a
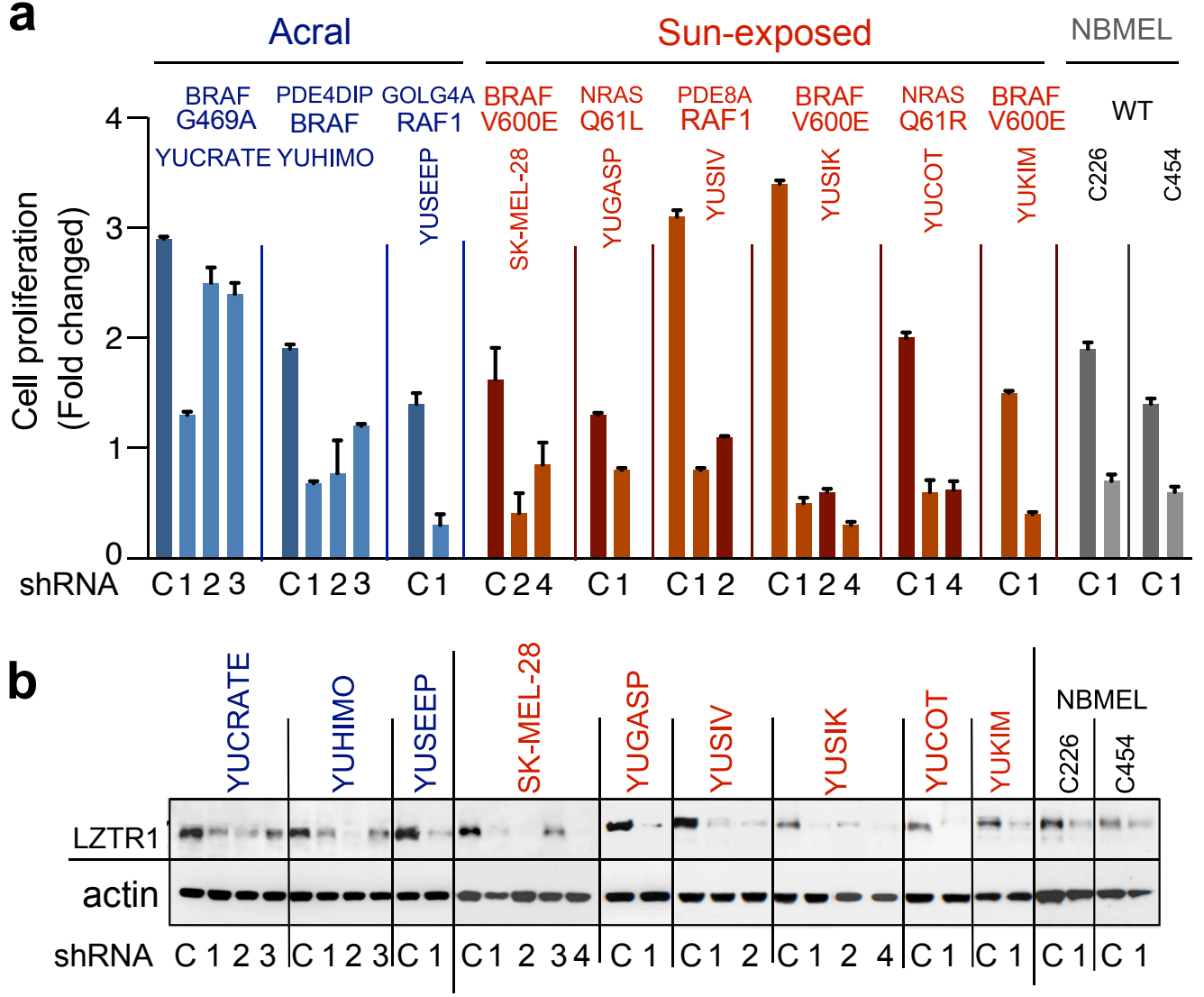

C

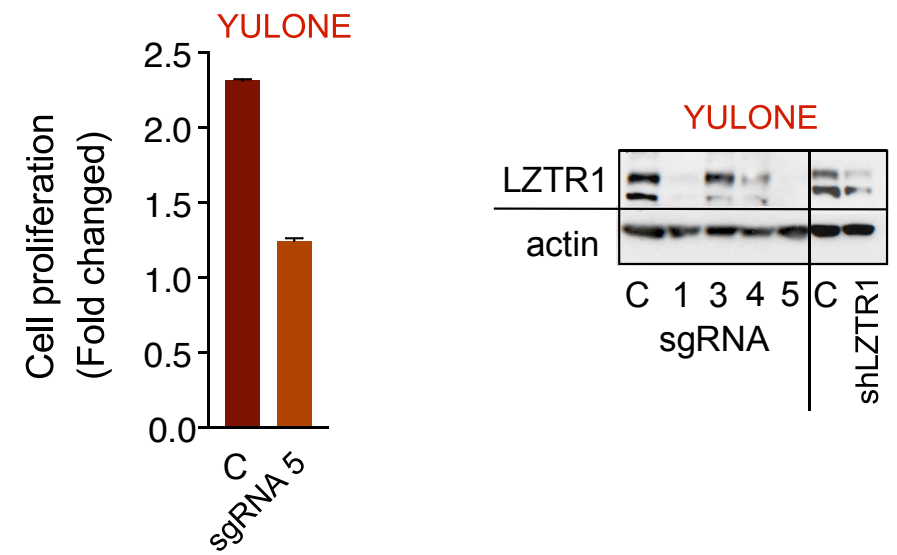

Figure 3: Cell proliferation in response to suppression of LZTR1. a, Impact of LZTR1 knockdown on cell proliferation in nine primary melanoma cell lines and two normal human melanocyte lines (NBMEL). Key mutations are indicated

(Supplementary Table 9). Bar plots depict fold change between the $3^{\text {rd }}$ and $6^{\text {th }}$ day after infection with LZTR1 shRNA (numbered), as compared to control (scrambled) shRNA ('C'). All shRNAs significantly reduced proliferation relative to control ( $P$ $<0.05$; two-sided $t$ test with unequal variance). b. Western blot showing the efficiency of LZTR1 knockdown in primary acral and sun-exposed melanoma cell lines, and in normal melanocytes, related to panel a. c, Cell proliferation (left) and LZTR1 expression (right) of a sun-exposed melanoma cell line that lost one LZTR1 allele in response to genomic modification by different CRISPR-Cas9 sgRNAs targeting 
LZTR1. Bar plots depict fold change between the $3^{\text {rd }}$ and $6^{\text {th }}$ day after infection with sgRNA 5. Reduced cell proliferation is statistically significant $(P<0.05$; two-sided $t$ test with unequal variance). Bars in a, c represent the mean of triplicate or quadruplet wells and error bars indicate SEM. Actin levels in b, c show protein loading. Cell lines are indicated above all plots in a-c and colored according to their origin: acral melanoma (blue), sun-exposed melanoma (red), normal melanocyte (grey). NBMEL, newborn melanocyte cultured from Caucasian foreskin; WT, wildtype. 
a
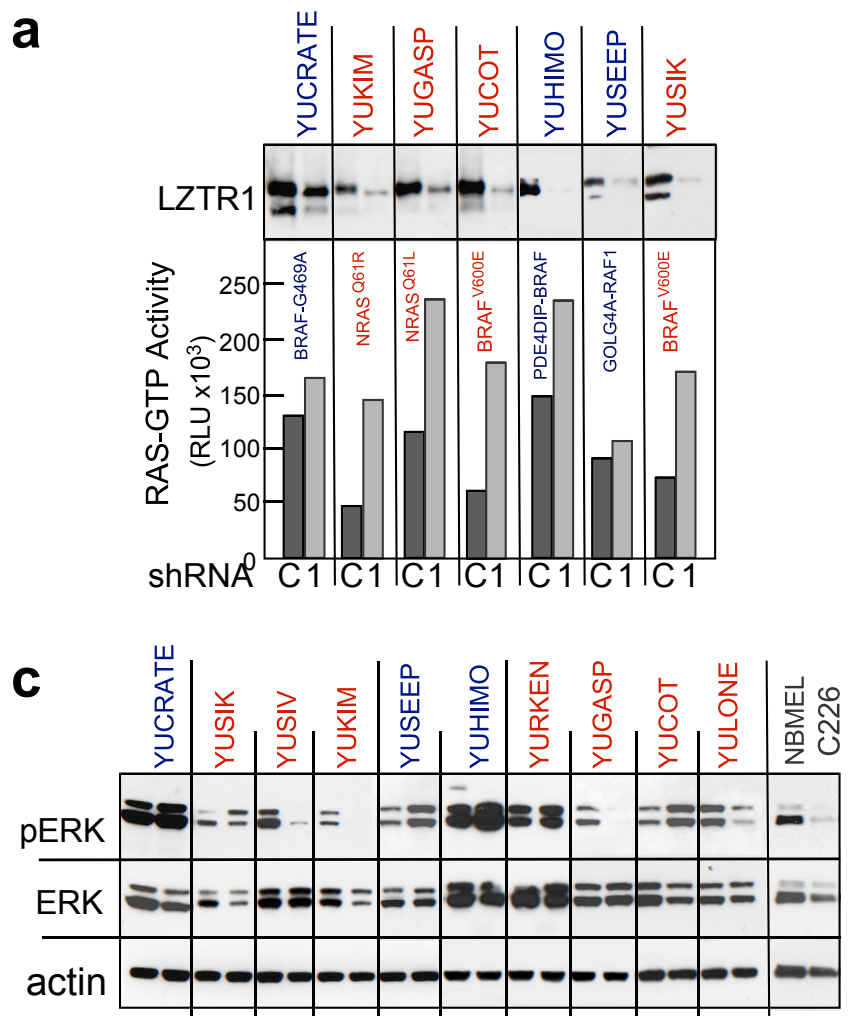

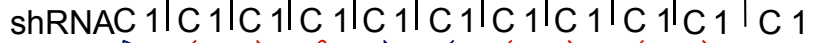
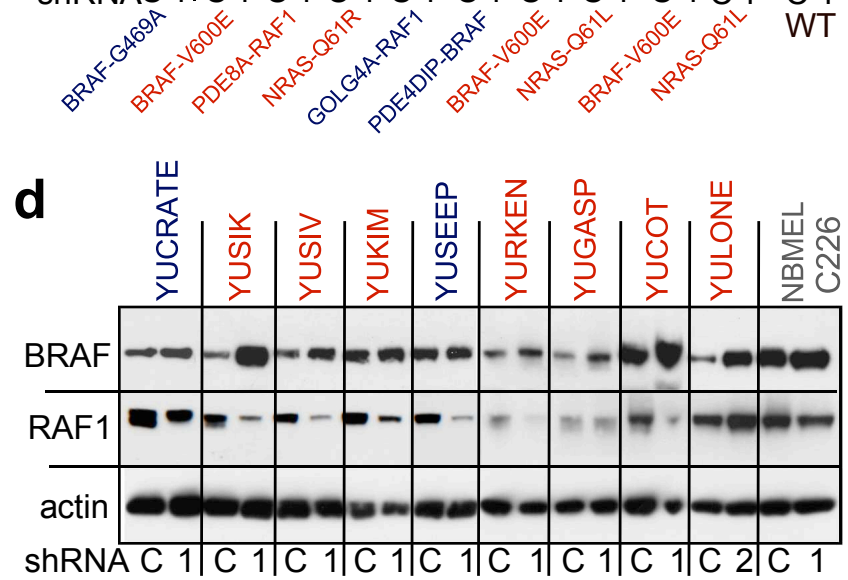

b

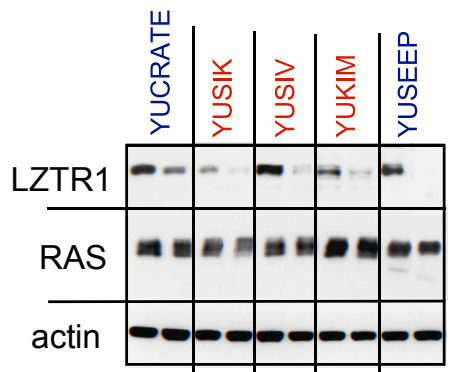
ShRNA C \begin{tabular}{l|l|l|l|l|l|l|l|l}
\hline & C & C & 1 & C & 1 & C & 1
\end{tabular}

e

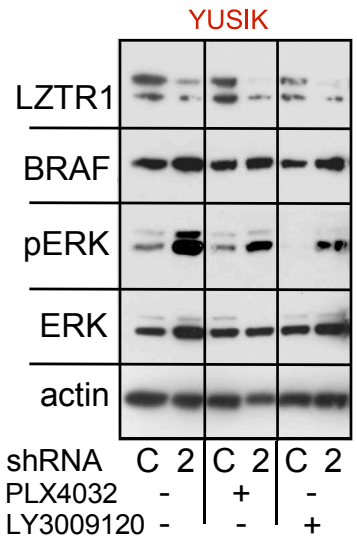

YUGASP

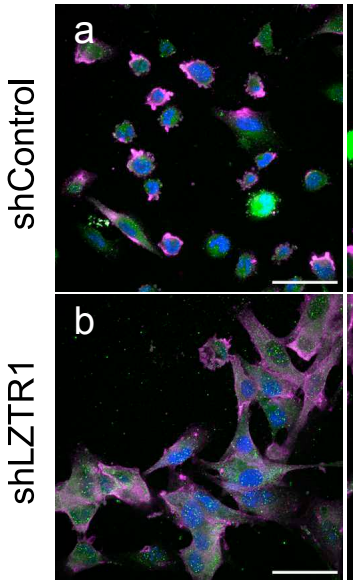

RAS GM130

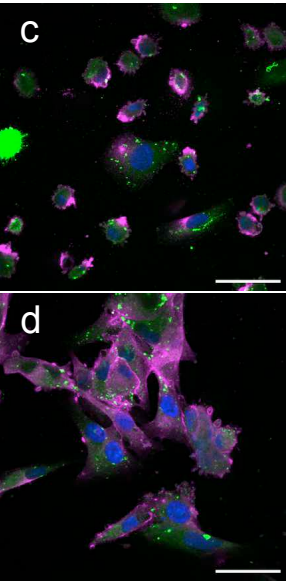

RAS Calnexin

Figure 4: Changes in MAPK signaling in response to LZTR1 knockdown. a, b, Impact of LZTR1 loss on (a) RAS-GTP activity as measured by RAS-GTPase Activation ELISA assay, and (b) RAS levels, five days after shLZTR1 infection. c, Effect of LZTR1 loss on MAPK activity. Key somatic events are indicated below. WT, wildtype. d, e Increased levels of BRAF activity in response to shLZTR1 is associated with increase pERK. In panel e, cells were incubated with RAF the kinase inhibitors PLX4032 (500 $\mathrm{nM}$ ) or LY3009120 (100 nM) for four hours at the end of treatment with shRNA. f, RAS translocation to the cytoplasm in response to shLZTR1; a-d, RAS is visualized by staining with magenta; Green (Cy2) indicates $\operatorname{GM} 130(a, b)$ and calnexin (c, d). Scale 
bar $=50 \mu \mathrm{m}$. Blue and red indicate acral and sun-exposed melanoma cell lines, respectively. shRNAs in a-e are indicated by numeric identifiers. C, scrambled shRNA control. Actin levels in a-e show protein loading. Cell lines are indicated above all plots in a-f and colored according to their origin: acral melanoma (blue), sun-exposed melanoma (red), normal melanocyte (grey). NBMEL, newborn melanocyte. 
a

Hallmark Pathways

MYC TARGETS V1

UNFŌLDED PROTTEIN_RESPONSE

MYC_TARGËTS_V2

G2M CHECKPOINT

DNA_REPAIR

Enriched in

low LZTR1 high LZTR1

Apoptosis $\longrightarrow$

IL6 JAK STAT3 SIGNALING INFLAMMATAORY RESPONSE

INTERFERON_GAMMMA_RESPONSE

TNFA SIGNALING VIA NFKB

ALLOGGRAFT_REJECTIŌN

C

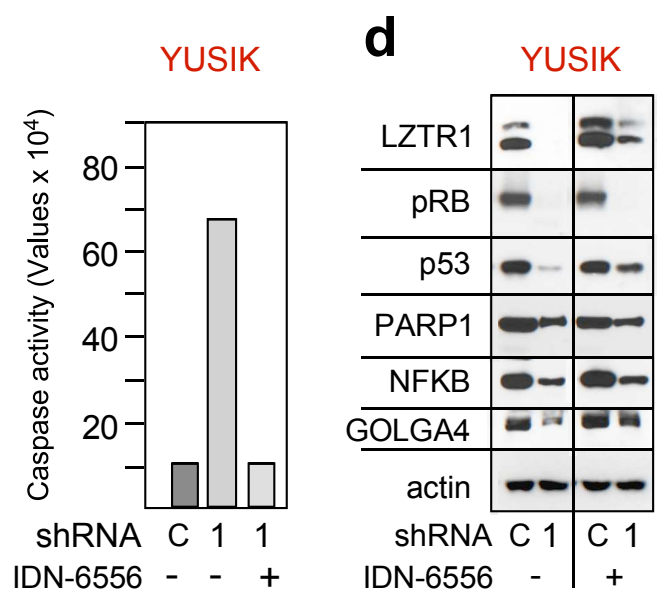

f

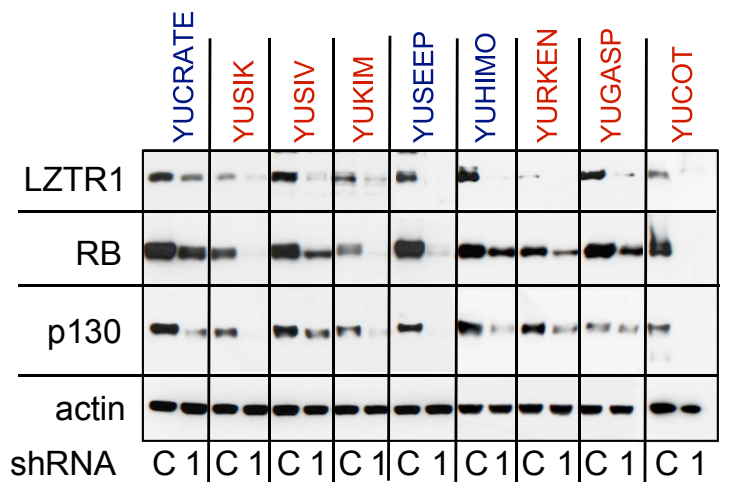

b

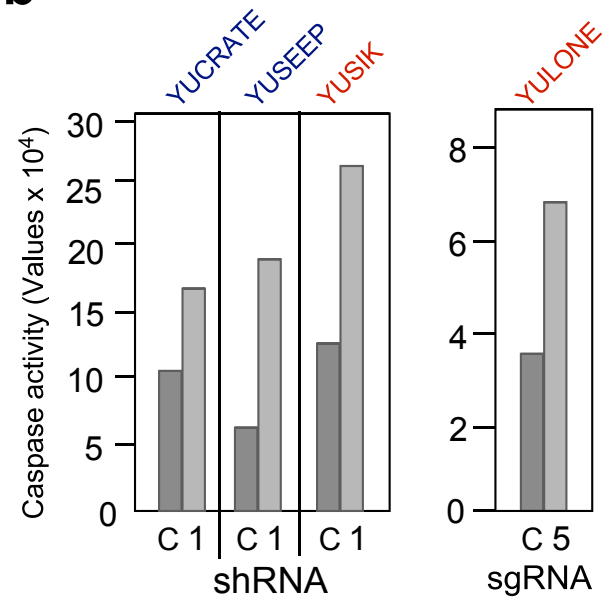

LZTR1 low

LZTR1 high

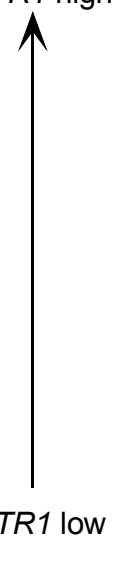

sgRNA

e

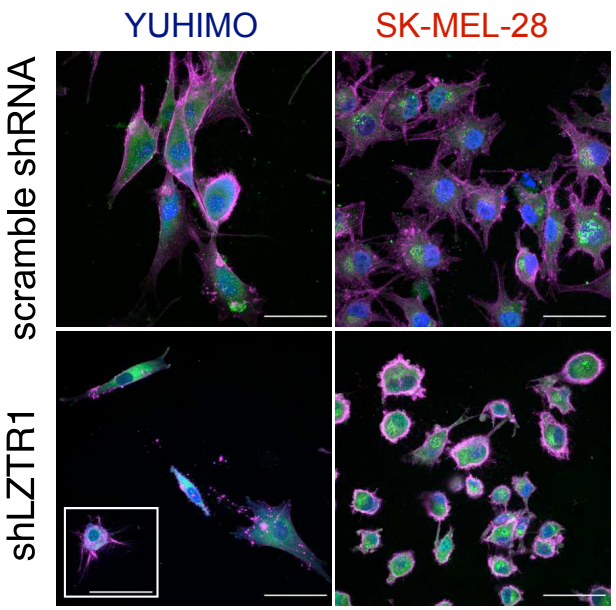

Actin GM130
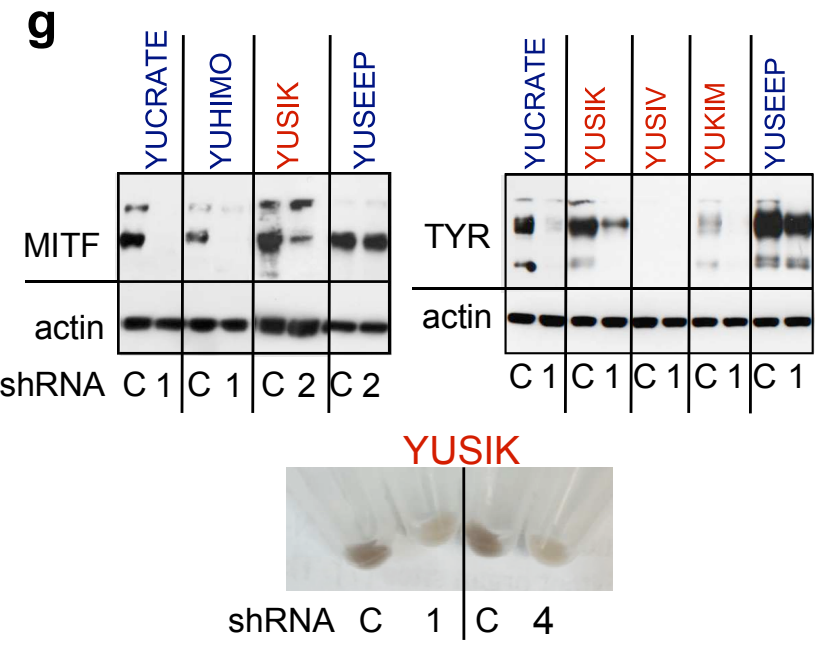

Figure 5: Impact of LZTR1 knockdown on apoptosis, Rb signaling, and pigmentation. a, Gene Set Enrichment Analysis (GSEA) ${ }^{94}$ showing concordance in hallmark pathways among bulk acral melanoma tumors, acral melanoma single-cell 
transcriptomes (scRNA-seq), and a primary melanoma cell line (LZTR1 vs. KD), in relation to high vs. low LZTR1 expression. Gold, high positive normalized enrichment score (NES); blue, high negative NES; KD, knockdown. b, LZTR1 shRNA and sgRNA (CRISPR-Cas9) induce apoptosis in melanoma cells. c, d, Effects of inhibiting caspase activity with IDN-6556 (IDN, $2 \mu \mathrm{M}, 3$ days). As shown in c, IDN-6556 suppressed shLZTR1-induced caspase activity. As shown in d, IDN-6556 increased the levels of LZTR1 (known to be degraded by caspases) and rescued caspase substrates, such as GOLGA4, p53, and to a lesser extent, NF-kB. e, Effect of shLZTR1 on cell morphology and actin filament organization. Actin filaments were visualized by staining with rhodamine-phalloidin (magenta) and the Golgi with anti-GM130 (green, Cy2). The nuclei are stained with DAPI (blue). Scale bar $=50 \mu \mathrm{m}$. f, shLZTR1 downregulates Rb and p130 (also known as RBL2). g-i, Impact of shLZTR1 on MITF (panel g), tyrosinase (TYR) (panel h), and pigmentation (panel i). shRNAs in b-d and f-i are indicated by unique numerical identifiers. $C$, scrambled shRNA control. Actin levels in $\mathbf{b}-\mathbf{d}$ and $\mathbf{f}-\mathbf{h}$ show protein loading. Of note, the actin control in $f$ and $h$ is identical because the same membrane was used to blot the proteins. Cell lines are indicated above all plots in $\mathbf{b}-\mathbf{g}$ and colored according to their origin: acral melanoma (blue), sun-exposed melanoma (red). 
a

Parental

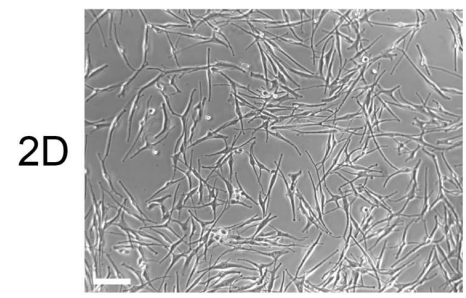

3D

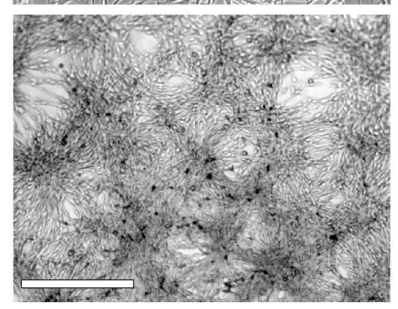

b

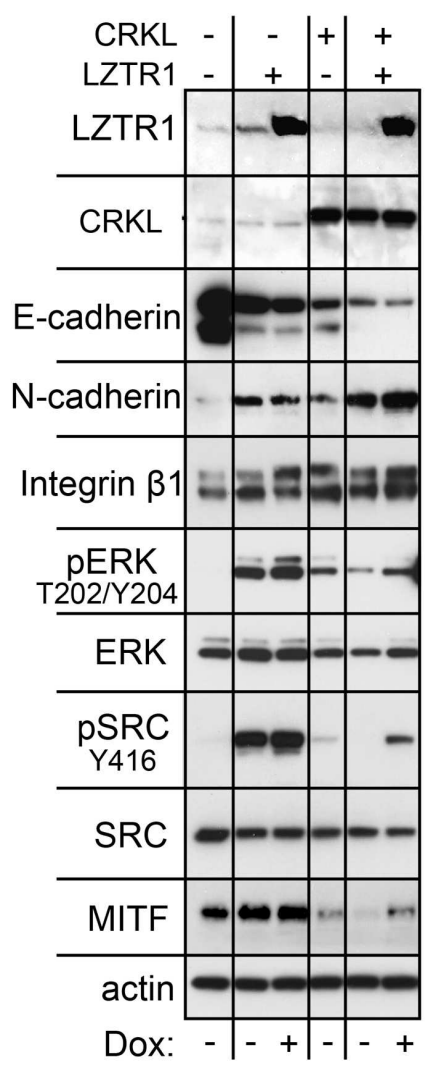

LZTR1
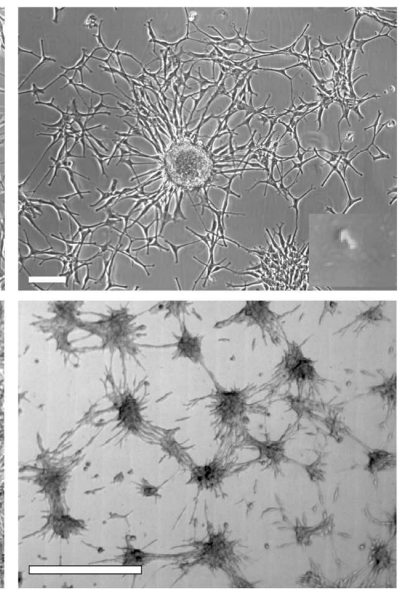

C

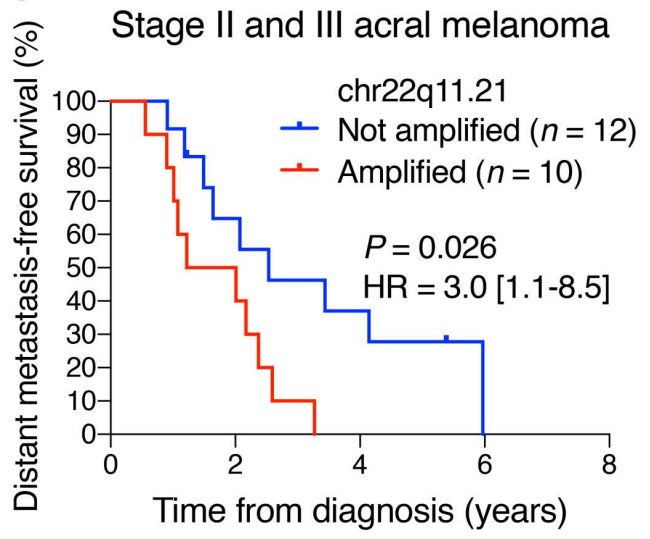

d

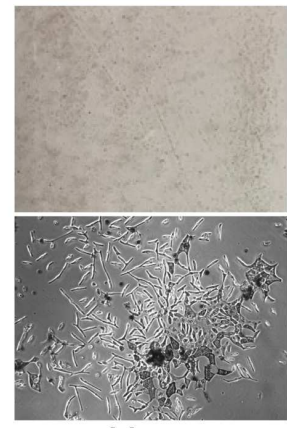

None
CRKL
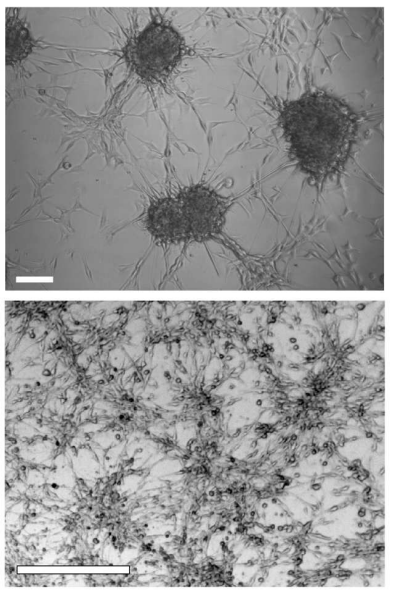

LZTR1+CRKL

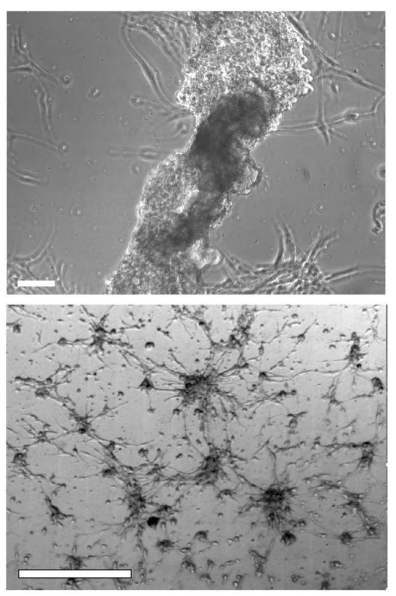

e

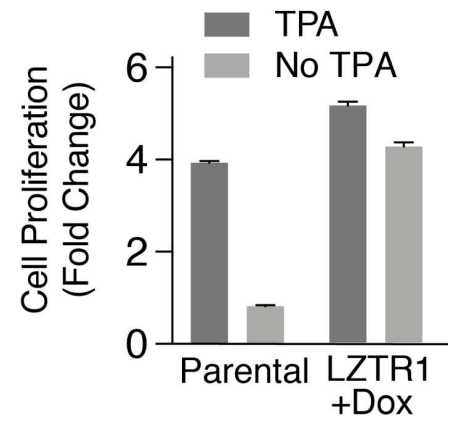

f

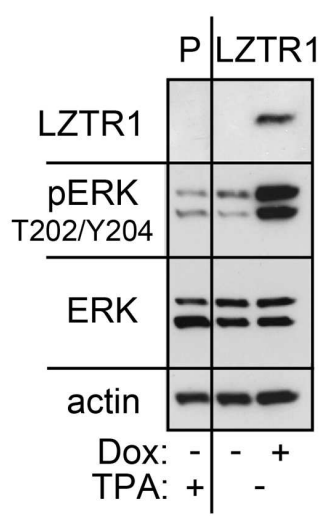

Figure 6: LZTR1 and CRKL confer properties consistent with malignant cell transformation and metastasis initiation. a, Morphological changes and spheroid formations in early passage normal human melanocytes (NBMEL C1220) overexpressing LZTR1 and/or CRKL. Top: Phase-contrast images of parental and infected cells in 2D culture. LZTR1 images were taken after two days induction with doxycycline (200 ng/ml), CRKL after three days of infection with PLX304-CRKL, and LZTR1+CRKL after six days infection of LZTR1 melanocytes with PLX304-CRKL and three days stimulation with doxycycline. Insert in LZTR1 shows that colonies were 
evident without magnification. The non-induced LZTR1 cells grow as a monolayer, as seen for the parental non-transformed melanocytes (Parental). Scale bar $=100 \mu \mathrm{m}$. Bottom: Low magnification images showing 3D cultures of melanocytes seeded in $0.5 \%$ collagen for three days. LZTR1 and CRKL, both alone and in combination, induced aggregation and multicellular spheroids. Scale bar $=500 \mu \mathrm{m}$. b. Western blot showing critical changes in normal human melanocytes overexpressing LZTR1, CRKL, or both (as seen in the top two lanes) compared to parental (-). Cells were harvested after incubation in regular medium, or medium supplemented with doxycycline for two days when applicable (Dox, $200 \mathrm{ng} / \mathrm{ml}$ ). Of note, an increase in LZTR1 produced by basal promoter activity was sufficient to induce constitutive MAPK and SRC activities. Actin levels show protein loading. c, Kaplan Meier plot showing differences in distant metastasis-free survival (DMFS) between acral melanoma patients stratified by 22q11.21-amplification status. Patients with stage II or III disease at diagnosis with available DMFS data are shown (Yale cohort). Statistical significance was assessed by a log-rank test. HR, hazard ratio. 95\% HR confidence interval is shown in brackets. d, 2D cultures of spontaneously immortalized mouse melanocytes (C57BL) forming colonies in the absence of TPA in response to LZTR1 (Dox). The dark colonies are seen without magnification (top), and under phase-contrast microscope (bottom). e, Cell proliferation of parental and LZTR1 transformed C57BL mouse melanocytes. f, Western blots displaying LZTR1 expression and MAPK activation (pERK) in TPA-starved (-) mouse melanocytes in response to doxycycline $(+, 200 \mathrm{ng} / \mathrm{ml})$, compared to parental, non-transformed cells $(P)$. 
Table 1: Patient characteristics and sequencing data

\begin{tabular}{lcc}
\hline Characteristics & $\begin{array}{c}\text { Acral } \\
(n=104)\end{array}$ & $\begin{array}{c}\text { Sun- } \\
\text { exposed } \\
(n=157)\end{array}$ \\
\hline Age (years) & & \\
$\quad$ Median (range) & $62(29-89)$ & $66(20-94)$ \\
Sex, $n(\%)$ & & \\
$\quad$ Female & $42(40)$ & $61(39)$ \\
$\quad$ Male & $62(60)$ & $96(61)$ \\
Stage at tumor resection, $n(\%)$ & & \\
0 & $1(1)$ & $0(0)$ \\
1 & $10(10)$ & $7(4)$ \\
2 & $39(38)$ & $22(14)$ \\
3 & $28(27)$ & $7(4)$ \\
4 & $26(25)$ & $121(77)$ \\
Tumor sample site, $n(\%)$ & & \\
$\quad$ Primary & $81(78)$ & $41(26)$ \\
Metastatic & $23(22)$ & $116(74)$ \\
WES, $n$ (\%) & & \\
Yale University & $32(33)$ & $134(100)$ \\
Central South University & $65(67)$ & $0(0)$ \\
Bulk RNA-seq, total $n(n$ with WES) & & $00(37)$ \\
Yale University & $22(17)$ & 0 \\
Central South University & $23(21)$ & \\
\hline
\end{tabular}

Out of 104 acral melanoma patients, 97 were profiled by WES, 7 were profiled by bulk RNA-seq and not WES, and 45 were profiled by both. Of 157 sun-exposed melanoma patients, 134 were profiled by WES, 23 were profiled by bulk RNA-seq and not WES, and 37 were profiled by both. 


\section{Methods}

\section{Human subjects}

All clinical specimens in this study were collected with informed consent for research use and were approved by the Yale University and Central South University Institutional Review Boards in accordance with the Declaration of Helsinki. Melanoma tumor specimens were excised to alleviate tumor burden. CSU samples were collected from Xiangya Hospital, Hospital for Skin Diseases (Institute of Dermatology), Chinese Academy of Medical Sciences in Nanjin, Third Affiliated Hospital of Sun Yat-sen University, Hunan Provincial Tumor Hospital, Xiangya Hospital, Central South University, First Affiliated Hospital of Harbin Medical University and Wuhan Union Hospital.

\section{Nucleic acids extraction}

Melanomas were sequenced from snap-frozen tumors (Yale and CSU cohorts) or low passage cell cultures (<4) as previously described ${ }^{2,4}$ (Supplementary Tables 1 and 8). DNA from melanoma cells and freshly frozen tumors was extracted with the DNeasy purification kit (Qiagen Inc., Valencia, CA). High melanin content was removed with OneStep ${ }^{\mathrm{TM}}$ PCR Inhibitor Removal Kit (Zymo Research Corporation, Irvine, CA). Directzol $^{\text {TM }}$ RNA MiniPrep w/ Zymo-Spin ${ }^{\mathrm{TM}}$ IIC Columns (Zymo cat \# D4019) were used to extract RNA from tumors, and the RNeasy PowerLyzer Tissue \& Cells Kit (Qiagen, CAT \# 15055-50) was used to extract RNA from peripheral blood mononuclear cells (PBMCs) and melanoma cells.

\section{Whole exome sequencing}

Sample preparation: The quality of genomic DNA was determined by estimating the $A_{260} / A_{280}$ and $A_{260} / A_{230}$ ratios by nanodrop, both of which required to be $>1.8$, and by electrophoresis in 1\% agarose gel in which high quality DNA migrates as a single high molecular weight band. One $\mu \mathrm{g}$ of genomic DNA was sheared to a mean fragment length of about 220 bp using focused acoustic energy (Covaris E220). The size distribution of the fragmented sample was determined by using the Caliper LabChip GX system. The fragmented DNA samples were transferred to a 96-well plate and library construction was completed using a liquid handling robot. Following fragmentation, we added T4 DNA polymerase and T4 polynucleotide kinase that blunt end and phosphorylate the fragments. The large Klenow fragment then adds a single adenine residue to the $3^{\prime}$ end of each fragment and custom adapters (IDT) are ligated using T4 DNA ligase. Magnetic AMPure XP beads (Beckman Coulter) were used to purify and size select the adapter-ligated DNA fragments. The adapter-ligated DNA fragments were then PCR amplified using custom-made primers (IDT). During PCR, a unique six base index was inserted at both ends of each DNA fragment. Sample concentration was determined by picogreen and the fragment length distribution using the Caliper LabChip GX system. Samples yielding at least $1 \mu \mathrm{g}$ of amplified DNA were used for capture.

Targeted capture and sequencing: For the CSU cohort, capture was performed using the NimbleGenSeqCap Med Exome 44M kit, followed by 151 bp paired-end sequencing on the Illumina HiSeq X 10 platform. TrimGalore (version 0.3.7) and FastQC (v0.11.2) 
were used to remove adapters and low-quality sequences from the raw data. For the Yale cohort, equal amounts of each sample were pooled prior to capture. Example: for 16 samples per lane $62.5 \mathrm{ng}$ of each genomic DNA library was pooled (1 $\mu \mathrm{g}$ total) and lyophilized with Cot-1 DNA and universal adapter blocking oligos (IDT). The dried sample was reconstituted according to the manufacturer's protocol (IDT), heatdenatured, and mixed with biotinylated DNA probes produced by IDT (xGen Exome Panel). Hybridizations were performed at $65^{\circ} \mathrm{C}$ for 16 hours. Once the capture was complete, the samples were mixed with streptavidin-coated beads and washed with a series of stringent buffers to remove non-specifically bound DNA fragments. The captured fragments were PCR amplified and purified with AMPure XP beads. Samples were quantified by qRT-PCR using a commercially available kit (KAPA Biosystems) and insert size distribution determined with the LabChip GX. Samples with a yield of $\geqslant 0.5$ $\mathrm{ng} / \mathrm{\mu l}$ were used for sequencing. Sample concentrations were normalized to $2 \mathrm{nM}$ and loaded onto Illumina NovaSeq 6000 flow cells at a concentration that yields at least $600 \mathrm{Gbp}$ of passing filter data per lane. Samples were sequenced using $101 \mathrm{bp}$ pairedend sequencing reads according to lllumina protocols.

\section{Bulk and single-cell RNA sequencing}

Bulk RNA-seq: For the CSU cohort, total RNA was depleted of rRNA using the RiboZero rRNA removal kit, namely, $1 \mu \mathrm{g}$ of total RNA was used as input for rRNA removal. Sequencing libraries were generated using the TruSeq RNA sample prep kit (Illumina). The libraries were sequenced as 151 bp paired-end reads using an Illumina HiSeq $X$ Ten platform. For the Yale cohort, rRNA was depleted starting from 25-1000ng of total RNA using the Kapa RNA HyperPrep Kit with RiboErase (KR1351). Indexed libraries that met appropriate cut-offs for both quantity and quality were quantified by qRT-PCR using a commercially available kit (KAPA Biosystems) and insert size distribution was determined with the LabChip GX or Agilent Bioanalyzer. Samples with a yield of $\geq 0.5$ $\mathrm{ng} / \mathrm{ul}$ were used for sequencing. Samples were run on a combination of Illumina HiSeq 2500, HiSeq 4000, and NovaSeq instruments, and multiplexed using unique dual barcode indexes (to avoid sample contamination or barcode hopping).

scRNA-seq: To obtain a single-cell transcriptional portrait of a chr22q11.21-amplified tumor, we analyzed a primary acral melanoma specimen (YUJASMIN, Yale cohort) with 6 focal copies of 22q11.21, as determined by WES (Supplementary Table 1). The 10x Chromium 5' expression profiling platform with V1 chemistry was applied to a cryopreserved tumor cell suspension from YUJASMIN sorted for viable singlets to target 10,000 cells. Cells were sorted in the following ratios prior to library preparation: $50 \%$ $\mathrm{CD}^{+} \mathrm{CD} 45^{+} \mathrm{T}$ cells: $25 \% \mathrm{CD}^{-}{ }^{-} \mathrm{CD} 45^{+}$non-T immune cells: $25 \% \mathrm{CD}^{-} 5^{-}$stromal/cancer cells. Cell viability was assessed by the LIVE/DEAD ${ }^{\text {TM }}$ Fixable Red Dead Cell Stain Kit (catalog \#L34971, Thermo Fisher). The following antibodies were used: Alexa Fluor ${ }^{\circledR}$ 488 anti-human CD45 antibody (clone H130, catalog \#304019, BioLegend); APC antihuman CD3 antibody (clone HIT3a, catalog \#300319, BioLegend). The 10x library was sequenced on an Illumina HiSeq 2500 instrument. 


\section{Tumor genotyping from whole exome sequencing data}

Sequencing reads from exome-captured samples were analyzed with a combination of germline and somatic variant calling, permitting the identification of somatic variants, loss-of-heterozygosity ( $\mathrm{LOH}$ ) regions and copy-number variation (CNV) regions.

SNVs and indels: BAM files of aligned reads were created for each sample by aligning the sequencing reads to the GRCh37 human reference with decoy sequences (the "hs37d5" reference) using BWA MEM ${ }^{95}$, marking duplicates using Picard MarkDuplicates (http://broadinstitute.github.io/picard), and then performing indel realignment and base quality score recalibration using GATK $v 3.2^{96}$. Then, variants were called using the tumor/normal bam files in three ways: 1) a joint variant call using GATK HaplotypeCaller, GenotypeGVCFs and hard filtering following GATK 3.2 best practices; 2) somatic SNP variant calls using MuTect with options "max_alt_alleles_in_normal_count $=6$ ", "max_alt_allele_in_normal_fraction $=0.1$ " and "max_alt_alleles_in_normal_qscore_sum $=200$ "; 3 ) somatic indel variant calls using Indelocator with options "minCoverage=6", "minNormalCoverage $=4$ " and "minFraction $=0.2$ ". The output from the three variant callers were merged using inhouse scripts into a single VCF file, containing the union of GATK variants and MuTect/Indelocator somatic variants, marking variants called as somatic by MuTect or Indelocator as "somatic".

Those variants were annotated using Annovar ${ }^{97}$ and VEP ${ }^{98}$, and then the somatic variants were filtered using the following criteria: 1) tumor alt depth $\geq 4,2$ ) normal read depth $\geq 4,3$ ) normal alt depth $\leq 1$ or normal alt frequency less than $1 / 5$ tumor alt frequency, 4) the maximum population frequency of the variant from ExAC ${ }^{99}, \mathrm{NHLBI}$, 1000 Genomes, or Yale Exome database must be less than $2 \%$ for a cancer-related gene (any gene in the Oncomine or Foundation Medicine gene panels or COSMIC CG Census gene list) or $1 \%$ for any other gene. Also, only protein changing variants with a VEP impact of MEDIUM or HIGH, or variants within 15 bases of a protein coding splice site were reported in the final output.

LOH: Loss-of-heterozygosity $(\mathrm{LOH})$ regions were identified using the joint variant calls generated from GATK. For each variant that was called heterozygous in the normal and had a depth $\geq 20$ in the normal, the allele frequency of the tumor and normal were subtracted ("abs (tumorAF - normalAF)"). Then the R loess and predict functions were used to smooth the allele frequency differences, and then any region with fitted values above 0.1 were identified as LOH. Tumor purity was estimated by taking the maximum mean of any LOH region, multiplied by 2 (as the tumorAF deviation, plus and minus, identifies the homozygous tumor proportion of the sample).

CNVs: Gene-level copy-number variant (CNV) regions were identified by first calculating the mean read depth for each RefGene coding exon, for the tumor and normal samples. Normalized tumor/normal read depth ratios were computed for each exon (normalized by the mean read depth of the tumor and normal across the exome), and then, using a partitioning of the genome into $20 \mathrm{~kb}$ bins, a mean ratio for each $20 \mathrm{~kb}$ region of the 
genome, which contains an exon, was computed. Those mean ratios were de-noised and segmented by circular binary segmentation (CBS) using the DNAcopy library from $\mathrm{R}$ (http://bioconductor.org/packages/release/bioc/html/DNAcopy.html.) Regions with a value deviating from the expected 1.0 ratio were identified as CNVs. For each CNV, ploidy is calculated using a deviation step value of 0.5 for high tumor purity samples (purity $\geq 80 \%$, a 0.4 step for purity between $40 \%$ and $80 \%$, and 0.32 step for purity less than $40 \%$ ), with the ploidy equaling 2 plus or minus the number of deviation steps.

Focal amplifications and deletions were identified with GISTIC2.0 (version 2.0.23, release date 27 Mar 2017) ${ }^{33}$ using the CBS segmentation files described above and the hg19 reference genome (GRCh37). No marker input file was provided. Parameters were specified according to the authors' recommended run profile: amplification and deletion thresholds were set to 0.1 , the q value threshold was set to 0.1 with a confidence level of 0.95 , and $\log _{2}$ ratios were capped at 1.5 . Gene-level GISTIC analysis and broad analysis were also applied, with a focal length cutoff of 0.7 . Wide peaks identified with a $Q$ value less than 0.1 in each melanoma subtype were aggregated into a master list (Supplementary Tables 3 and 4), and genes within each wide peak were used to construct a copy number matrix. Of note, if two or more peaks were identified within the same cytoband, we appended a suffix to the cytoband name to denote the melanoma subtype in which the cytoband was identified (AC, acral; SE, sun-exposed). If more than one peak was identified within the same cytoband for a given melanoma subtype, the subtype acronym was followed by a numerical identifier $(1,2$, etc.). For cases in which one peak completely encompassed another one and where both peaks had the same orientation (i.e., amplified or deleted), the shorter one was eliminated.

For comparative genomics and survival analyses, we constructed a matrix containing the mean copy number per wide peak (rows) for each melanoma tumor sample (columns). The mean copy number per wide peak was calculated as the average genelevel copy number (estimated as described above) per wide peak. We subtracted 2 from all gene-level values so that copy number-neutral regions are equal to 0 (Supplementary Data).

\section{Visualization of somatic alterations across patients}

Recurrently mutated genes and significant focal CNVs were visualized using the Oncoprint function in ComplexHeatmap ${ }^{100}$. The default bar plot (top) was replaced with a bar plot showing the number of nonsynonymous SNVs and indels per patient. For CNV regions, amplifications and deletions were calculated by averaging the GISTICgenerated gene-level copy numbers for all genes within each wide peak. Peaks with an average copy number above 3 (one additional copy) were deemed amplified, whereas peaks with an average copy number below 1.4 were considered deleted.

\section{Bulk RNA-seq analysis}

Raw RNA-seq reads were aligned with Salmon ${ }^{101}$ (version 0.99) to the GENCODE v. $25^{102}$ reference transcript assembly. Subsequently, the tximport ${ }^{103}$ was used to generate an expression matrix normalized to transcripts per million (TPM). Protein- 
coding genes were determined using Ensembl release 92 human annotation ${ }^{104}$ (GRCh38.p12, Apr 2018), extracted by biomaRt ${ }^{105}$ (version 2.40.5) and non-proteincoding genes were omitted. Expression values were renormalized to TPM after this step. For batch normalization, we applied ComBat from sva ${ }^{106}$ using a parametric adjustment for sequencing center and year of sequencing. Following batch correction, negative values were replaced with zero and the expression matrix was $\log _{2}-$ transformed after adding a pseudo-count of 1.

To delineate pathways associated with focal amplification of 22q11.21 (Fig. 2d), genes differentially expressed between 22q11.21 amplified and non-amplified acral melanomas were identified by constructing a linear model ( $/ m$ function in R) to predict amplification status as a function of 1 ) gene expression ( $\log _{2}$ adjusted) and 2) tumor stage (at the time of resection). The $t$ value corresponding to the expression vector of each gene was used to rank-order the transcriptome. Pre-ranked gene set enrichment analysis (GSEA) ${ }^{107}$ was subsequently applied to the ranked-ordered transcriptome in order to assess HALLMARK pathways in MSigDB (version 7.2) ${ }^{108}$.

Related to Fig. 2e, we curated a list of immunomodulatory genes, including immune checkpoint molecules, and analyzed their expression in both acral cohorts using hierarchical clustering applied with Pearson correlation and Ward D2.

\section{Gene fusion detection from RNA-seq data}

To identify fusion genes, we aligned the RNA-seq reads for each sample to the GRCh38 human reference genome using HISAT2 ${ }^{109}$. Candidate fusion transcripts in the sequencing reads were identified with STAR-Fusion, employing the STAR aligner and FusionInspector annotator to identify the position of the chimeric RNA. For ease of manual review, the fusions were sub-grouped, with each fusion placed into the first group that either gene matched: 1) mitochondrial genes, 2) immunoglobulin genes, 3) protocadherin genes, 4) commonly expressed fusions using GTEx expression data, 5) fusions of neighboring/local_rearrangement genes, 6) non-annotated genes, and 7) all others i.e., the rare, non-local fusions of annotated protein coding genes.

\section{Survival analysis}

Cox proportional hazards regression was applied to estimate overall survival associations. Cases with an initial diagnosis preceding the sequenced tumor by more than 5 years were excluded from analysis $(n=5)$. To estimate stage-adjusted associations with overall survival, stage was added as a covariate. Kaplan-Meier plots for comparison of survival curves were generated either by the survminer ${ }^{110}$ package in $\mathrm{R}$ (version 0.4 .5 ) or by Graphpad Prism (version 8).

To determine survival associations of focal CNVs identified by GISTIC (Fig. 2a, Supplementary Fig. 3a, Supplementary Table 6), we applied Cox regression separately to each region within each acral melanoma cohort using the copy number matrix described above (see Copy number analysis). In all cases, we dichotomized each CNV by analyzing amplified $(>0)$ versus non-amplified $(\leq 0)$ and deleted $(<0)$ 
versus non-deleted $(\geq 0)$. Survival z-scores were combined across cohorts using Stouffer's method ${ }^{111}$, yielding an unweighted meta-z-score for each gene.

To analyze SNV- and indel-related survival associations in acral melanomas, we examined genes harboring one or more nonsynonymous mutations with at least $5 \%$ recurrence frequency in either acral melanoma cohort. These data were used to create a binary matrix in which recurrently mutated genes were rows and patients were columns (1, at least one recurrent SNV or indel; 0 , otherwise). Survival z-scores were combined across cohorts as indicated above. The following four genes were insufficiently recurrent in at least one cohort to run Cox regression: CGREF1, NF1, TP53, and ARID2. To calculate survival associations for these genes, we randomly upsampled patients from the Yale cohort in order to match the size of the CSU cohort. We then generated a cross-cohort survival Z-score for each gene (Supplementary Table 6).

To relate LZTR1 expression to overall survival, we dichotomized patients in each cohort by determining an expression threshold that discriminates 22q11.21-amplified from nonamplified patients at a defined specificity. This was done to link the threshold for dichotomization with 22q11.21 amplification without being confounded by the upper range of LZTR1 expression in non-amplified tumors. We used a specificity cutpoint of $95 \%$ for acral melanomas profiled in this study and sun-exposed melanomas profiled by TCGA. A specificity cutpoint of $90 \%$ was used for sun-exposed melanomas profiled in this work owing to a lack of evaluable samples in the 'high' group $(n=1)$ at a specificity cutpoint of $95 \%$. Notably, LZTR1 expression was also significantly associated with adverse outcomes when assessed as a continuous variable (i.e., without dichotomization) in sun-exposed melanomas profiled by TCGA $(Z=2.86, P=0.004)$. Skin cutaneous melanoma (SKCM) expression, copy number, and survival data from TCGA were downloaded from cBioPortal ${ }^{87}$.

\section{Single-cell RNA sequencing analysis}

Single-cell RNA-seq reads were mapped to the GRCh38 human reference assembly and barcode-deduplicated using Cell Ranger (version 3.0.2). In total, 7,551 cells were sequenced, yielding a median of 945 genes per cell. The expression matrix generated by Cell Ranger was converted to counts per million (CPM) and was $\log _{2}$ transformed after addition of a pseudo-count to every value. Cells with less than 500 expressed genes were removed before importing the data into Seurat ${ }^{112}$ (version 3.0.2). Seurat was applied for pre-processing (default settings), data normalization (default settings), identifying the most variable features, dimension reduction (PCA and UMAP), finding marker genes, and clustering the single-cell expression data. In finding variable features, the low and high mean cutoffs were set to 0.0125 and 3 , respectively, and the dispersion cutoffs were respectively set to 0.5 and infinity, with 20 bins. PCA was generated on the most variable genes with the first 15 principal components. Neighbors were calculated from the first 13 PCA components (as determined by the JackStraw function), followed by cluster analysis (resolution $=0.5$ ). 
Single-cell copy number analysis: To estimate large-scale copy number alterations from scRNA-seq data, we used CONICSmat (COpy-Number analysis In single-Cell RNASequencing from an expression matrix) ${ }^{36}$ as implemented in the $\mathrm{R}$ package, CONICSmat. Per the authors' recommendations, raw read counts were divided by 10 and were $\log _{2}$-adjusted after adding one to each count value. Chromosome arm positions from GRCh38 were used to define arm coordinates. Briefly, genes expressed in 5 cells or less were filtered, a normalization factor for each cell was calculated, and a Gaussian mixture model was calculated based on the z-score of the average centered gene expression for each region across all cells. Melanoma cells $(n=321)$ were split into two groups based on the estimated relative copy number in the $22 q$ arm. Cells with a normalized copy number between 0 and 0.10 were used for normalization of the expression data. Histograms were generated using plotHistogram and by setting the zscore threshold to 4. Chromosomal alterations in each single cell were visualized by plotHistogramHeatmap with the authors' recommended parameters (window size $=120$, expression threshold $=0.2$, visualization threshold $=1$ ). Of 12 genes identified by GISTIC within the chr22q11.21 wide peak (Supplementary Table 3), relative copies of 22q11.21 for each cell were calculated by averaging the estimated copy number for all 7 genes with detectable expression $(\mathrm{CPM}>0)$.

To characterize the RNA expression profile associated with chr22q11.21 focal amplification, the median relative copy number (0.45) inferred by CONICSmat was used to split the cells into chr22q11.21 high and low groups. The $\log _{2}$-adjusted scRNA-seq dataset normalized by Census ${ }^{113}$ was compared between these two groups to identify differentially expressed genes using a two sided $t$-test with unequal variance, and the resulting $t$-statistics were used for ranking the gene list. This gene list was submitted to pre-ranked GSEA ${ }^{107}$ to interrogate 50 HALLMARK pathways (1,000 permutations, weighted enrichment statistics, MSigDB version $7.2^{108}$ ).

Single-cell differentiation status: To predict the relative differentiation status of each melanoma cell profiled by scRNA-seq, we used CytoTRACE ${ }^{42}$, a computational framework for inferring developmental potential on the basis of transcriptional diversity. Each of the 321 acral melanoma cells from chr22q11.21 amplified tissue received a CytoTRACE score between 0 (more differentiated) and 1 (less differentiated). CytoTRACE scores were visualized by Uniform Manifold Approximation and Projection (UMAP). To determine whether CytoTRACE is influenced by genes located on 22q, we reran CytoTRACE after excluding all genes on the 22q arm (Supplementary Fig. 4e). 
Short hairpin RNA (shRNA), CRISPR-Cas9 sgRNA, and cell viability tests

We used puromycin-bearing MISSION lentiviral vectors pLKO.1 shRNA to test the effect of downregulation of target proteins on cell proliferation and signal transduction, employing scramble vector SHOO2 as a negative control (MISSION, Sigma-Aldrich, Supplementary Table 8), or scrambled RNA (Supplementary Table 8). LentiCRISPRv2 plasmid was obtained from Addgene (addgene.org). Guide sequences targeting LZTR1 (Supplementary Table 8) were designed using CHOPCHOP (https://chopchop.cbu.uib.no/) and cloned into LentiCRISPRv2 to generate single sgRNA carrying plasmids following a standard method ${ }^{114}$. A non-target sequence was included as the control (Supplementary Table 8).

The plasmids were packaged in lentiviral vectors with ViraPower ${ }^{\mathrm{TM}}$ Lentiviral Packaging Mix kit (Thermo Fisher, cat \# K497500), and transfected into 293T cells. The medium was collected and filtered with Millex-GV $33 \mathrm{~mm}$ PVDF filter (Millipore SLGV033RS) and then concentrated with Amicon Ultra-15 centrifugal filters (Millipore UFC910024). Melanoma cells and normal human melanocytes were infected with the lentiviruses, medium was changed the following day, and the cells were then incubated with puromycin $(2.5 \mu \mathrm{g} / \mathrm{ml})$ for five days. Cells were collected and processed for western blotting with antibodies to target proteins. In addition, two days after infection the shRNA treated cells were seeded in 96-well plates in triplicate or quadruplet wells and tested for cell viability in the absence and presence of puromycin for $72 \mathrm{hrs}$ with the CellTiterGlo® Luminescent Cell Viability Assay, for apoptosis or RAS activity GTPase assay.

Alternatively, GV298-U6-MCS-Ubiquitin-Cherry-IRES-puromycin lentiviral plasmids were purchased from GeneChem, China. The plasmids were co-transfected with packaging plasmids (pspAX2 and pMD2G) into 293T cells using Turbofect (Thermo Scientific) according to the manufacturer's instructions. Lentiviruses were collected after 48 and 72 hours and used to infect into acral melanoma cells. Infected cells were incubated in medium supplemented with puromycin $(1 \mu \mathrm{g} / \mathrm{ml})$, for two or three days, seeded in $96-$ well plates ( $2 \times 10^{3} /$ well, five replicates) and cell viability was measured with Cell Counting Kit-8 (CCK-8) (Bimake.com, China). The CCK-8 test was repeated every 24 hrs for three days.

\section{CRKL and LZTR1 lentivirus vectors}

pDONR223-CRKL and pDONR223-LZTR1 were purchased from Addgene and DNASU, respectively. LZTR1 was transferred into plnducer20 vector ${ }^{115}$ (a gift from $\mathrm{Dr}$. Thomas F. Westbrook, Baylor College of Medicine), and CRKL to PLX304 with the Gateway LR Clonase II Enzyme mix (Thermo Fisher 11791020). The identity of each vector was validated by targeted sequencing. Lentiviruses were produced as described above and the plnducer20 LZTR1 infected melanocytes were selected with $250 \mathrm{mg} / \mathrm{ml}$ Geneticin (G418) from American Bio (Canton, MA), CAT \# AB05057-05000), and PLX304CRKL with 2.5 $\mu \mathrm{g} / \mathrm{ml}$ Blasticidin S HCl from Thermo Fisher Scientific (Waltham, MA), CAT \# A1113903. 


\section{Cell proliferation and apoptosis}

The melanoma cells were grown in OptiMEM (Invitrogen, Carlsbad, CA) supplemented with $5 \%$ fetal calf serum and antibiotics. Normal human melanocytes (NBMEL) were grown from newborn foreskins in medium supplemented with bFGF, heparin, IBMX and dbcAMP ${ }^{76}$. Mouse melanocytes were grown from one-day old newborn pups in the presence of horse serum, TPA, melanotropin, isobutyl methyl xanthine, and placental extract. They became immortalized and were shifted to medium containing only TPA after $\sim 20$ passages in cultures ${ }^{116}$. Some of the Yale melanoma cell lines were characterized by next-generation sequencing before ${ }^{2,4}$ (Supplementary Table 9).

Cell proliferation was measured with the CellTiter-Glo ${ }^{\circ}$ Luminescent Cell Viability Assay (Promega Corporation, Madison, WI). Melanoma cells were seeded in 96-well plates in triplicate or quadruplet wells after knockdown with hairpin lentivirus shRNA as indicated. Standard Error (SE) was calculated employing GraphPad Prism 7 software ${ }^{117}$. In addition, we seeded cells in 12-well plates (10-15,000/well) and measured proliferation by counting the number of cells from triplicate wells over a period up to 7-9 days with Beckman Cell Counter. For cell count by crystal violet, we seeded cells $\left(3 \times 10^{3} /\right.$ well) in 6 -well plates and then incubated for 10 days. Following incubation, cells were immobilized with $4 \%$ paraformaldehyde in PBS for 15 min, stained with crystal violet for 10 min and then washed with PBS. A minimum of three random fields at $40 \mathrm{X}$ magnification were counted to determine cell numbers. Each sample had three replicates.

The rate of apoptosis was measured using the Dead Cell Apoptosis Kit with Alexa Fluor ${ }^{\circledR} 488$ annexin $V$ and propidium iodide (Invitrogen, V13241) following manufacturer instructions.

PLX4032 (500 nM, Plexxikon) $)^{117}$ or LY3009120 (100 nM, Selleck, Pittsburgh, PA, Catalog No.S7842) were added to the growth medium 4 hours before harvesting the cells for Western blotting.

For 3D cultures, melanocytes were suspended in $1 \mathrm{ml}$ medium and seeded on $0.5 \%$ collagen (Cultrex, R\&D Systems, Minneapolis, MN, Cat \# 3442-050-01), in 24 well plates for three days.

\section{Microscopy}

Images were acquired using an inverted Nikon Eclipse Ti fluorescence microscope with a Plan Apochromat lambda 60X/1.40 Oil objective or a Plan Fluor 4X/0.13 objective for fluorescent images or DIC images, respectively, a CSU-W1 confocal spinning disk unit, an iXon Ultra 888 camera (Andor Technology), MLC 400B laser unit (Agilent Technologies) and NIS Elements software (Nikon).

\section{Western blotting and antibodies}

We used western blots to identify the levels of proteins as previously described ${ }^{69}$. Cell extracts $(20 \mu \mathrm{g} / \mathrm{lane})$ were fractionated in $3 \%-8 \%$ or $4-12 \%$ tris-acetate gel (NP0006, NuPAGE Life Technologies). The membranes were probed with the primary antibodies 
described in Supplementary Table 10. All antibodies were used at the concentrations recommended by the manufacturers.

\section{RAS activity assay}

The amount of GTP-bound RAS was determined using the Ras GTPase Chemi ELISA Kit (Active Motif North America, 1914 Palomar Oaks Way, Carlsbad, C 92008) following the manufacturer's protocol. Melanoma cells treated with control shRNA or shLZTR1 were collected five days after infection by scraping on ice, washed with cold PBS, lysed, centrifuged and $50 \mu \mathrm{g}$ protein/assay, in triplicates, were used following the manufacturer instructions.

\section{Immunostaining}

Cells were grown on the surface of 4-well slides, washed 2-3 times with PBS, fixed with $4 \%$ paraformaldehyde for $15 \mathrm{~min}$ at room temperature, washed three times with PBS, permeabilize with $0.2 \%$ NP40 in PBS for 5 minutes, washed with PBS and incubate in PBS containing $1 \%$ BSA or (blocking buffer) for one hour. The cells were incubated with anti-GM130 antibody (clone 4A3 Millipore, Mouse), or calnexin (mouse mAb) for $1 \mathrm{hr}$ at room temperature, and stained with secondary Alexa Fluor (Cy2) diluted in blocking buffer 1:1000 for $1 \mathrm{hr}$. They were washed 3X with PBS, incubated with rhodaminephalloidin to stain actin and DAPI to stain the nucleus.

\section{Statistical analysis}

Linear relationships were modeled by linear regression $\left(R^{2}\right)$, and a $t$ test was used to assess whether the result was significantly nonzero. When data were normally distributed, group comparisons were determined using a $t$ test with unequal variance or a paired $t$ test, as appropriate; otherwise, a Wilcoxon test was applied. Results with $P<$ 0.05 were considered significant. Data analyses were performed with $R$ and Prism v7 (GraphPad Software, Inc.). The investigators were not blinded to allocation during experiments and outcome assessment. No sample-size estimates were performed to ensure adequate power to detect a pre-specified effect size.

\section{Data availability}

Raw sequencing data will be deposited in the Sequence Read Archive (SRA) and https://www.biosino.org/node/ and expression data will be deposited in the Gene Expression Omnibus (GEO). Accession numbers are pending. 


\section{References}

1. Curtin, J.A. et al. Distinct sets of genetic alterations in melanoma. $N$ Engl J Med 353, 2135-47 (2005).

2. Krauthammer, M. et al. Exome sequencing identifies recurrent somatic RAC1 mutations in melanoma. Nat Genet 44, 1006-14 (2012).

3. Hodis, E. et al. A landscape of driver mutations in melanoma. Cell 150, 251-63 (2012).

4. Krauthammer, M. et al. Exome sequencing identifies recurrent mutations in NF1 and RASopathy genes in sun-exposed melanomas. Nat Genet 47, 996-1002 (2015).

5. Network, T.C.G.A. Genomic Classification of Cutaneous Melanoma. Cell 161, 1681-1696 (2015).

6. Zhang, T., Dutton-Regester, K., Brown, K.M. \& Hayward, N.K. The genomic landscape of cutaneous melanoma. Pigment Cell Melanoma Res 29, 266-83 (2016).

7. Bastian, B.C. et al. Gene amplifications characterize acral melanoma and permit the detection of occult tumor cells in the surrounding skin. Cancer Res 60, 19681973 (2000).

8. Bastian, B.C., Olshen, A.B., LeBoit, P.E. \& Pinkel, D. Classifying melanocytic tumors based on DNA copy number changes. Am J Pathol 163, 1765-70 (2003).

9. Furney, S.J. et al. Genomic characterisation of acral melanoma cell lines. Pigment Cell Melanoma Res 25, 488-92 (2012).

10. Kong, Y. et al. Analysis of mTOR Gene Aberrations in Melanoma Patients and Evaluation of Their Sensitivity to PI3K-AKT-mTOR Pathway Inhibitors. Clin Cancer Res 22, 1018-27 (2016).

11. Liang, W.S. et al. Integrated genomic analyses reveal frequent TERT aberrations in acral melanoma. Genome Res 27, 524-532 (2017).

12. Kong, Y. et al. Frequent Genetic Aberrations in the CDK4 Pathway in Acral Melanoma Indicate the Potential for CDK4/6 Inhibitors in Targeted Therapy. Clin Cancer Res 23, 6946-6957 (2017).

13. Hayward, N.K. et al. Whole-genome landscapes of major melanoma subtypes. Nature 545, 175-180 (2017).

14. Yeh, I. et al. Targeted Genomic Profiling of Acral Melanoma. J Natl Cancer Inst 111, 1068-1077 (2019).

15. Newell, F. et al. Whole-genome sequencing of acral melanoma reveals genomic complexity and diversity. Nature Communication 11, 5259 (2020).

16. Park, C.K. \& Kim, S.K. Clinicopathological significance of intratumoral and peritumoral lymphocytes and lymphocyte score based on the histologic subtypes of cutaneous melanoma. Oncotarget 8, 14759-14769 (2017).

17. Klemen, N.D. et al. Survival after checkpoint inhibitors for metastatic acral, mucosal and uveal melanoma. J Immunother Cancer 8(2020).

18. Curtin, J.A., Busam, K., Pinkel, D. \& Bastian, B.C. Somatic activation of KIT in distinct subtypes of melanoma. J Clin Oncol 24, 4340-6 (2006).

19. Beadling, C. et al. KIT Gene Mutations and Copy Number in Melanoma Subtypes. Clin Cancer Res 14, 6821-8 (2008). 
20. Vazquez Vde, L. et al. Molecular profiling, including TERT promoter mutations, of acral lentiginous melanomas. Melanoma Res 26, 93-9 (2016).

21. Shim, J.H. et al. Mutational profiling of acral melanomas in Korean populations. Exp Dermatol 26, 883-888 (2017).

22. Moon, K.R. et al. Genetic Alterations in Primary Acral Melanoma and Acral Melanocytic Nevus in Korea: Common Mutated Genes Show Distinct Cytomorphological Features. J Invest Dermatol 138, 933-945 (2018).

23. Zaremba, A. et al. Clinical and genetic analysis of melanomas arising in acral sites. Eur J Cancer 119, 66-76 (2019).

24. Puig-Butille, J.A. et al. Genetic alterations in RAS-regulated pathway in acral lentiginous melanoma. Exp Dermatol 22, 148-50 (2013).

25. Yu, S. et al. TERT copy gain predicts the outcome of high-dose interferon alpha2b therapy in acral melanoma. Onco Targets Ther 11, 4097-4104 (2018).

26. Furney, S.J. et al. The mutational burden of acral melanoma revealed by wholegenome sequencing and comparative analysis. Pigment Cell Melanoma Res 27, 835-8 (2014).

27. Shi, K. et al. Distinct Genomic Features in a Retrospective Cohort of Mucosal, Acral and Vulvovaginal Melanomas. J Am Acad Dermatol (2019).

28. Behbahani, S., Malerba, S. \& Samie, F.H. Racial and ethnic differences in the clinical presentation and outcomes of acral lentiginous melanoma. British Journal of Dermatology $\mathbf{n} / \mathbf{a}$.

29. Bradford, P.T., Goldstein, A.M., McMaster, M.L. \& Tucker, M.A. Acral lentiginous melanoma: incidence and survival patterns in the United States, 1986-2005. Arch Dermatol 145, 427-34 (2009).

30. Smit, N.P. et al. Increased melanogenesis is a risk factor for oxidative DNA damage--study on cultured melanocytes and atypical nevus cells. Photochem Photobiol 84, 550-5 (2008).

31. Walker, G.J. et al. Virtually $100 \%$ of melanoma cell lines harbor alterations at the DNA level within CDKN2A, CDKN2B, or one of their downstream targets. Genes Chromosomes Cancer 22, 157-63 (1998).

32. Jönsson, G. et al. Genomic profiling of malignant melanoma using tilingresolution arrayCGH. Oncogene 26, 4738-48 (2007).

33. Mermel, C.H. et al. GISTIC2.0 facilitates sensitive and confident localization of the targets of focal somatic copy-number alteration in human cancers. Genome Biol 12, R41 (2011).

34. Subramanian, A. et al. Gene set enrichment analysis: a knowledge-based approach for interpreting genome-wide expression profiles. Proc Natl Acad Sci U $S$ A 102, 15545-50 (2005).

35. Madden, E., Logue, S.E., Healy, S.J., Manie, S. \& Samali, A. The role of the unfolded protein response in cancer progression: From oncogenesis to chemoresistance. Biol Cell 111, 1-17 (2019).

36. Müller, S., Cho, A., Liu, S.J., Lim, D.A. \& Diaz, A. CONICS integrates scRNA-seq with DNA sequencing to map gene expression to tumor sub-clones.

Bioinformatics 34, 3217-3219 (2018).

37. Intlekofer, A.M. \& Finley, L.W.S. Metabolic signatures of cancer cells and stem cells. Nat Metab 1, 177-188 (2019). 
38. Schatton, T. et al. Identification of cells initiating human melanomas. Nature $\mathbf{4 5 1}$, 345-9 (2008).

39. Abbaszadegan, M.R. et al. Isolation, identification, and characterization of cancer stem cells: A review. J Cell Physio/ 232, 2008-2018 (2017).

40. Eun, K., Ham, S.W. \& Kim, H. Cancer stem cell heterogeneity: origin and new perspectives on CSC targeting. BMB Rep 50, 117-125 (2017).

41. Thankamony, A.P., Saxena, K., Murali, R., Jolly, M.K. \& Nair, R. Cancer Stem Cell Plasticity - A Deadly Deal. Front Mol Biosci 7, 79 (2020).

42. Gulati, G.S. et al. Single-cell transcriptional diversity is a hallmark of developmental potential. Science 367, 405-411 (2020).

43. Frattini, V. et al. The integrated landscape of driver genomic alterations in glioblastoma. Nat Genet 45, 1141-9 (2013).

44. Chen, R.H. Cullin 3 and Its Role in Tumorigenesis. Adv Exp Med Biol 1217, 187210 (2020).

45. Pathmanaban, O.N. et al. Association of Genetic Predisposition With Solitary Schwannoma or Meningioma in Children and Young Adults. JAMA Neurol 74, 1123-1129 (2017).

46. Evans, D.G. et al. Schwannomatosis: a genetic and epidemiological study. $J$ Neurol Neurosurg Psychiatry 89, 1215-1219 (2018).

47. Luo, L.Y. \& Hahn, W.C. Oncogenic Signaling Adaptor Proteins. J Genet Genomics 42, 521-529 (2015).

48. Cheung, H.W. et al. Amplification of CRKL induces transformation and epidermal growth factor receptor inhibitor resistance in human non-small cell lung cancers. Cancer Discov 1, 608-25 (2011).

49. Kim, Y.H. et al. Genomic and functional analysis identifies CRKL as an oncogene amplified in lung cancer. Oncogene 29, 1421-30 (2010).

50. Kostrzewska-Poczekaj, M. et al. Copy number gains of the putative CRKL oncogene in laryngeal squamous cell carcinoma result in strong nuclear expression of the protein and influence cell proliferation and migration. Sci Rep 10, 24 (2020).

51. Bigenzahn, J.W. et al. LZTR1 is a regulator of RAS ubiquitination and signaling. Science 362, 1171-1177 (2018).

52. Steklov, M. et al. Mutations in LZTR1 drive human disease by dysregulating RAS ubiquitination. Science 362, 1177-1182 (2018).

53. Umeki, I. et al. Delineation of LZTR1 mutation-positive patients with Noonan syndrome and identification of LZTR1 binding to RAF1-PPP1CB complexes. Hum Genet 138, 21-35 (2019).

54. Motta, M. et al. Dominant Noonan syndrome-causing LZTR1 mutations specifically affect the Kelch domain substrate-recognition surface and enhance RAS-MAPK signaling. Hum Mol Genet 28, 1007-1022 (2019).

55. Li, X. et al. Molecular and phenotypic spectrum of Noonan syndrome in Chinese patients. Clin Genet 96, 290-299 (2019).

56. Guemes, M. et al. LZTR1: Genotype Expansion in Noonan Syndrome. Horm Res Paediatr, 1-7 (2019).

57. Abe, T. et al. LZTR1 facilitates polyubiquitination and degradation of RASGTPases. Cell Death Differ 27, 1023-1035 (2020). 
58. Julien, O. \& Wells, J.A. Caspases and their substrates. Cell Death Differ 24, 1380-1389 (2017).

59. Nacak, T.G., Leptien, K., Fellner, D., Augustin, H.G. \& Kroll, J. The BTB-kelch protein LZTR-1 is a novel Golgi protein that is degraded upon induction of apoptosis. J Biol Chem 281, 5065-71 (2006).

60. Mukherjee, S., Chiu, R., Leung, S.M. \& Shields, D. Fragmentation of the Golgi apparatus: an early apoptotic event independent of the cytoskeleton. Traffic 8, 369-78 (2007).

61. Sengupta, S. \& Henry, R.W. Regulation of the retinoblastoma-E2F pathway by the ubiquitin-proteasome system. Biochim Biophys Acta 1849, 1289-97 (2015).

62. Li, B., Gordon, G.M., Du, C.H., Xu, J. \& Du, W. Specific killing of Rb mutant cancer cells by inactivating TSC2. Cancer Cell 17, 469-80 (2010).

63. Nicolay, B.N. et al. Proteomic analysis of $\mathrm{pRb}$ loss highlights a signature of decreased mitochondrial oxidative phosphorylation. Genes Dev 29, 1875-89 (2015).

64. Dyson, N.J. RB1: a prototype tumor suppressor and an enigma. Genes Dev 30, 1492-502 (2016).

65. Dick, F.A., Goodrich, D.W., Sage, J. \& Dyson, N.J. Non-canonical functions of the RB protein in cancer. Nat Rev Cancer 18, 442-451 (2018).

66. Fisher, D.E. Microphthalmia: a signal responsive transcriptional regulator in development. Pigment Cell Res 8, 145-149 (2000).

67. Wu, M. et al. c-Kit triggers dual phosphorylations, which couple activation and degradation of the essential melanocyte factor Mi. Genes Dev 14, 301-12 (2000).

68. Phung, B. et al. KITD816V Induces SRC-Mediated Tyrosine Phosphorylation of MITF and Altered Transcription Program in Melanoma. Mol Cancer Res 15, 1265-1274 (2017).

69. Halaban, R. et al. A novel anti-melanoma SRC-family kinase inhibitor. Oncotarget 10, 2237-2251 (2019).

70. Lin, F. et al. CRKL promotes lung cancer cell invasion through ERK-MMP9 pathway. Mol Carcinog 54 Suppl 1, E35-44 (2015).

71. Franke, F.C. et al. Novel role for CRK adaptor proteins as essential components of SRC/FAK signaling for epithelial-mesenchymal transition and colorectal cancer aggressiveness. Int J Cancer (2020).

72. Bates, R.C., Edwards, N.S. \& Yates, J.D. Spheroids and cell survival. Crit Rev Oncol Hematol 36, 61-74 (2000).

73. Haass, N.K., Smalley, K.S., Li, L. \& Herlyn, M. Adhesion, migration and communication in melanocytes and melanoma. Pigment Cell Res 18, 150-9 (2005).

74. Chaudhuri, O., Cooper-White, J., Janmey, P.A., Mooney, D.J. \& Shenoy, V.B. Effects of extracellular matrix viscoelasticity on cellular behaviour. Nature 584, 535-546 (2020).

75. Arozarena, I. \& Wellbrock, C. Phenotype plasticity as enabler of melanoma progression and therapy resistance. Nat Rev Cancer 19, 377-391 (2019).

76. Halaban, R. Culture of melanocytes from normal, benign and malignant lesions. in Culture of Human Tumor Cells (ed. Freshney, R.I., Pfranger, R.) 289-318 (John Wiley \& Sons, Inc, New York, NY, 2004). 
77. Halaban, R., Cheng, E., Zhang, Y., Mandigo, C.E. \& Miglarese, M.R. Release of cell cycle constraints in mouse melanocytes by overexpressed mutant E2F1E132, but not by deletion of $\mathrm{p} 16^{\mathrm{INK} 4 \mathrm{~A}}$ or p21 WAF1/CIP1. Oncogene 16, 2489-24501 (1998).

78. Wang, Y., Zhao, Y. \& Ma, S. Racial differences in six major subtypes of melanoma: descriptive epidemiology. BMC Cancer 16, 691 (2016).

79. Lv, J., Dai, B., Kong, Y., Shen, X. \& Kong, J. Acral Melanoma in Chinese: A Clinicopathological and Prognostic Study of 142 cases. Sci Rep 6, 31432 (2016).

80. De Wet, J., Tod, B., Visser, W.I., Jordaan, H.F. \& Schneider, J.W. Clinical and pathological features of acral melanoma in a South African population: A retrospective study. S Afr Med J 108, 777-781 (2018).

81. Wada, M. et al. Acral lentiginous melanoma versus other melanoma: A singlecenter analysis in Japan. J Dermatol 44, 932-938 (2017).

82. Sheen, Y.S. et al. A clinicopathological analysis of 153 acral melanomas and the relevance of mechanical stress. Sci Rep 7, 5564 (2017).

83. Desai, A., Ugorji, R. \& Khachemoune, A. Acral melanoma foot lesions. Part 1: epidemiology, aetiology, and molecular pathology. Clin Exp Dermatol 42, 845848 (2017).

84. Madankumar, R. et al. Acral melanocytic lesions in the United States: Prevalence, awareness, and dermoscopic patterns in skin-of-color and nonHispanic white patients. J Am Acad Dermatol 74, 724-30.e1 (2016).

85. Johnston, J.J. et al. Autosomal recessive Noonan syndrome associated with biallelic LZTR1 variants. Genet Med 20, 1175-1185 (2018).

86. Dhanoa, B.S., Cogliati, T., Satish, A.G., Bruford, E.A. \& Friedman, J.S. Update on the Kelch-like (KLHL) gene family. Hum Genomics 7, 13 (2013).

87. Cerami, E. et al. The cBio cancer genomics portal: an open platform for exploring multidimensional cancer genomics data. Cancer Discov 2, 401-4 (2012).

88. Gao, J. et al. Integrative analysis of complex cancer genomics and clinical profiles using the cBioPortal. Sci Signal 6, pl1 (2013).

89. McGary, E.C., Lev, D.C. \& Bar-Eli, M. Cellular adhesion pathways and metastatic potential of human melanoma. Cancer Biol Ther 1, 459-65 (2002).

90. Rathinam, R., Berrier, A. \& Alahari, S.K. Role of Rho GTPases and their regulators in cancer progression. Front Biosci 17, 2561-71 (2011).

91. Ridley, A.J. et al. Cell migration: integrating signals from front to back. Science 302, 1704-9 (2003).

92. Tate, J.G. et al. COSMIC: the Catalogue Of Somatic Mutations In Cancer. Nucleic Acids Res 47, D941-d947 (2019).

93. Sondka, Z. et al. The COSMIC Cancer Gene Census: describing genetic dysfunction across all human cancers. Nat Rev Cancer 18, 696-705 (2018).

94. Subramanian, A. et al. Gene set enrichment analysis: a knowledge-based approach for interpreting genome-wide expression profiles. Proc Natl Acad Sci USA 102, 15545-50 (2005).

95. Li, H. \& Durbin, R. Fast and accurate short read alignment with Burrows-Wheeler transform. Bioinformatics 25, 1754-60 (2009).

96. McKenna, A. et al. The Genome Analysis Toolkit: a MapReduce framework for analyzing next-generation DNA sequencing data. Genome Res 20, 1297-303 (2010). 
97. Wang, K., Li, M. \& Hakonarson, H. ANNOVAR: functional annotation of genetic variants from high-throughput sequencing data. Nucleic Acids Res 38, e164 (2010).

98. McLaren, W. et al. The Ensembl Variant Effect Predictor. Genome Biol 17, 122 (2016).

99. Lek, M. et al. Analysis of protein-coding genetic variation in 60,706 humans. Nature 536, 285-91 (2016).

100. Gu, Z., Eils, R. \& Schlesner, M. Complex heatmaps reveal patterns and correlations in multidimensional genomic data. Bioinformatics 32, 2847-9 (2016).

101. Patro, R., Duggal, G., Love, M.I., Irizarry, R.A. \& Kingsford, C. Salmon provides fast and bias-aware quantification of transcript expression. Nat Methods 14, 417419 (2017).

102. Frankish, A. et al. GENCODE reference annotation for the human and mouse genomes. Nucleic Acids Res 47, D766-d773 (2019).

103. Soneson, C., Love, M.I. \& Robinson, M.D. Differential analyses for RNA-seq: transcript-level estimates improve gene-level inferences. F1000Res 4, 1521 (2015).

104. Cunningham, F. et al. Ensembl 2019. Nucleic Acids Research 47, D745-D751 (2018).

105. Durinck, S., Spellman, P.T., Birney, E. \& Huber, W. Mapping identifiers for the integration of genomic datasets with the R/Bioconductor package biomaRt. Nature protocols 4, 1184 (2009).

106. Leek, J.T., Johnson, W.E., Parker, H.S., Jaffe, A.E. \& Storey, J.D. The sva package for removing batch effects and other unwanted variation in highthroughput experiments. Bioinformatics 28, 882-883 (2012).

107. Subramanian, A. et al. Gene set enrichment analysis: A knowledge-based approach for interpreting genome-wide expression profiles. Proceedings of the National Academy of Sciences 102, 15545-15550 (2005).

108. Liberzon, A. et al. The Molecular Signatures Database (MSigDB) hallmark gene set collection. Cell systems 1, 417-425 (2015).

109. Kim, D., Langmead, B. \& Salzberg, S.L. HISAT: a fast spliced aligner with low memory requirements. Nat Methods 12, 357-60 (2015).

110. Alboukadel, K., Marcin, K. \& Przemyslaw, B. Survminer: drawing survival curves using'ggplot2'. R package version 0.4.5. (2019).

111. Stouffer, S.A., Suchman, E.A., Devinney, L.C., Star, S.A. \& Williams Jr, R.M. The American soldier: Adjustment during army life. (Studies in social psychology in World War II), Vol. 1, xii, 599-xii, 599 (Princeton Univ. Press, Oxford, England, 1949).

112. Stuart, T. et al. Comprehensive Integration of Single-Cell Data. Cell 177, 18881902.e21 (2019).

113. Qiu, X. et al. Single-cell mRNA quantification and differential analysis with Census. Nature methods 14, 309-315 (2017).

114. Ran, F.A. et al. Genome engineering using the CRISPR-Cas9 system. Nat Protoc 8, 2281-2308 (2013).

115. Meerbrey, K.L. et al. The pINDUCER lentiviral toolkit for inducible RNA interference in vitro and in vivo. Proc Natl Acad Sci U S A 108, 3665-70 (2011). 
116. Tamura, A. et al. Normal murine melanocytes in culture. In Vitro Cellular \& Developmental Biology 23, 519-522 (1987).

117. Halaban, R. et al. PLX4032, a selective BRAF(V600E) kinase inhibitor, activates the ERK pathway and enhances cell migration and proliferation of BRAF(WT) melanoma cells. Pigment Cell Melanoma Res 23, 190-200 (2010). 


\section{Supplementary Files}

This is a list of supplementary files associated with this preprint. Click to download.

- LZTR1SuppFigs20201117v1.pdf

- LZTR1SuppTables20201115v1.xIsb

- LZTR1SuppData20201109v1.xlsb 